\title{
NeuroImage
}

\section{Flexible cognitive control: Effects of individual differences and brief practice on a complex cognitive task}

\author{
A.M. Clare Kelly, ${ }^{\mathrm{a}}$ Robert Hester, ${ }^{\mathrm{a}, \mathrm{b}}$ John J. Foxe, ${ }^{\mathrm{c}, \mathrm{d}}$ Marina Shpaner, ${ }^{\mathrm{c}, \mathrm{d}}$ and Hugh Garavan ${ }^{\mathrm{a}, \mathrm{d}, \mathrm{e}, *}$ \\ ${ }^{\mathrm{a} S}$ School of Psychology and Trinity College Institute of Neuroscience, Trinity College Dublin, Dublin 2, Ireland \\ ${ }^{\mathrm{b}}$ Cognitive Neuroscience Laboratory, Department of Psychology, University of Melbourne, Melbourne, Australia \\ ${ }^{\mathrm{c}}$ Program in Cognitive Neuroscience, Department of Psychology, City College of the City University of New York, New York, NY 10031, USA \\ ${ }^{\mathrm{d}}$ The Cognitive Neurophysiology Laboratory, Nathan S. Kline Institute for Psychiatric Research, \\ Program in Cognitive Neuroscience and Schizophrenia, Orangeburg, NY 10962, USA \\ e Department of Psychiatry and Behavioral Medicine, Medical College of Wisconsin, Milwaukee, WI 53226, USA
}

Received 17 September 2005; revised 6 January 2006; accepted 9 January 2006

Available online 7 March 2006

Brain activations underlying cognitive processes are subject to modulation as a result of increasing cognitive demands, individual differences, and practice. The present study investigated these modulatory effects in a cognitive control task which required inhibition of prepotent responses based on the contents of working memory (WM) and which enabled a novel dissociation of item-specific and taskskill effects resulting from brief practice. Distinct responses in areas underlying WM and inhibitory control in the absence of behavioral changes reflected different effects of item repetition and general task practice on tonic working memory and phasic inhibitory processes. Item repetition was associated with decreases in both unique and common areas subserving WM and inhibitory control. In contrast, general task practice was reflected in decreases in the level of tonic WM activity required to maintain a consistently high level of task performance but increased activity in a number of core inhibitory regions including dorsolateral and inferior $\mathrm{PFC}$ and inferior parietal cortex. Furthermore, both practice and individual differences in task performance were associated with the ability to modulate and maintain activity in frontostriatal areas mediating attentional control, suggesting that the areas that differ between individuals can be modulated by practice within an individual. These results raise the possibility that a fundamental human ability, reflexive cognitive control, is amenable to practice.

(C) 2006 Elsevier Inc. All rights reserved.

Keywords: Working memory; Inhibition; Flexible cognitive control; Interference; Practice; Performance effects

\footnotetext{
* Corresponding author. School of Psychology, Trinity College Dublin, Dublin 2, Ireland. Fax: +35316712006.

E-mail address: Hugh.Garavan@tcd.ie (H. Garavan).

Available online on ScienceDirect (www.sciencedirect.com).
}

\section{Introduction}

A defining feature of cognitive control is that it is reflexivewe can rapidly and flexibly adjust attentional processes and executive control over our thoughts and behavior in response to changing internal or external demands. This ability represents one of the most sophisticated capabilities of the human species (Braver et al., 2003). Current conceptualizations of working memory (WM) place it at the center of this flexible cognitive capacity. According to Kane, Engle and their colleagues (e.g, Conway et al., 2003; Engle et al., 1999; Engle and Kane, 2004; Kane and Engle, 2002; Kane et al., 2004), measures of WM capacity reflect "executive attention" - the ability to maintain goal-relevant information in a highly active and accessible state under conditions of interference or competition, a concept akin to the supervisory attentional system of Norman and Shallice (1986), the central executive of Baddeley and Hitch's (1974) conceptualization of WM, as well as concepts such as controlled attention (Schneider and Shiffrin, 1977), or the anterior attention system (Posner and Petersen, 1990). According to this framework, individual differences in WM capacity, as indexed by performance on WM span tasks, directly reflect individual differences in the ability to exert executive control over attention. Consistent with this, individual differences in performance on tasks requiring inhibitory control, such as the Stroop, antisaccade and dichotic listening tasks, or mnemonic tasks involving interference, have been observed to correlate with an individual's WM capacity (Bunting et al., 2004; Conway et al., 2001; Kane and Engle, 2000, 2003; Kane et al., 2001; Unsworth et al., 2004). Furthermore, the concept of executive attention has been linked to prefrontal cortical function and individual differences in the ability to modulate prefrontal activation when faced with demands on cognitive control (e.g., Bunge et al., 2001; Duncan, 2003; Kane and Engle, 2002; Mecklinger et al., 2003).

In a complementary line of research, a number of studies have demonstrated that increasing WM load can have a negative effect on attentional control, as reflected in performance on a range of 
tasks requiring inhibition of interfering stimuli or responses (e.g., Baddeley et al., 2001; de Fockert et al., 2001; Hester and Garavan, 2005; Roberts et al., 1994). These studies support the hypothesis that WM and inhibition share a central control or attentional mechanism, so that increased demand on WM negatively impacts on the attentional resources available to devote to inhibitory control. Neuroimaging evidence provides further support for a common neural basis for these abilities - for example, manipulating interference and load within a WM task was observed to activate broadly overlapping cortical networks involving PFC, insula, parietal cortex, and cerebellum (Bunge et al., 2001).

In a recent study, Hester et al. (2004b) further investigated the neural bases of the relationship between WM and inhibitory control. Taking into consideration that the decline in inhibitory control resulting from increases in WM load may be due to these processes sharing a common attentional network, they assessed how the brain succeeds in exerting inhibitory control as the number of items being rehearsed in WM increases.

Using event-related functional MRI, Hester et al. examined the BOLD response during performance of a task designed to integrate the requirement to actively maintain items in WM and GO/NOGO inhibitory performance. The Working Memory Inhibition (WMI) task involved a Sternberg verbal WM task, of varying loads (1-5 targets), embedded in a primary GO/NOGO task. Participants were first presented a target set of letter stimuli, followed by a stream of letters to which they had to respond if the letter was not a member of the memory set (GO trials), and withhold if the letter was a member of the memory set (NOGO trials). Thus, participants had to use the contents of WM in order to successfully perform the inhibition task.

Hester et al. observed that increasing WM load had a significant negative influence on inhibitory control, reducing the number of successful inhibitions to NOGO stimuli. However, while maintaining successful inhibitory control under increasing WM demands increased activation in regions common to both tasks, the predominant brain response was increased activations in those areas uniquely activated by the inhibitory demands of the task, including right dorsolateral prefrontal cortex, anterior cingulate cortex(ACC)/pre-supplementary motor area (pre-SMA), as well as in left prefrontal, temporal, and thalamic regions. The implication is that, while increased demand on common control resources might explain performance declines associated with high working memory loads, increased activation in task-specific areas underlies the ability to maintain successful inhibitory control despite high WM demands. Furthermore, Hester et al. observed that individual differences in the ability to modulate activity in some of these taskspecific areas (dIPFC and ACC/pre-SMA) were associated with performance differences in inhibitory control under high WM demands. This study demonstrated how flexible modulation of activation in both unique and shared cortical regions underlies the ability to maintain top-down control in response to increasing cognitive demands, and further, how individual differences play a significant role in this flexible modulation.

In the present paper, we ask: if dynamic and flexible modulation of activation in the brain enables the maintenance of successful control of attention under demanding conditions as shown by Hester et al., how might this response be affected by repeated task experience? That is, how might practice affect cognitive control under conditions of high WM demand?

The effects of task practice and repeated task experience on performance and brain activations, particularly in complex tasks which require the flexible modulation of brain activity, have been a focus of research interest in recent years. Practice on cognitive tasks is typically associated with changes in functional activations that may be characterized as a reorganization of functional activations, a redistribution of activation within the task network, or a generalized decrease in activation, the latter being thought to reflect increased efficiency in the underlying network and a more precise functional circuit (see Kelly and Garavan, 2005 for a review). Specifically, practice on WM tasks has been shown to decrease task demands, improve behavioral performance, and produce activation decreases in task-relevant areas (e.g., Garavan et al., 2000; Gevins et al., 1997; McEvoy et al., 1998; Jansma et al., 2001; Landau et al., 2004). To our knowledge, however, no study has yet investigated the effects of practice on the functional anatomy of a GO/NOGO inhibitory task, nor has it been investigated how practice might influence the ability to exert topdown cognitive control under conditions of high working memory demand.

In order to address this gap in the literature, we examined the effects of brief $(\sim 30 \mathrm{~min})$ practice on the WMI task. We were interested in how tonically active areas underlying verbal working memory processes and areas subserving phasic inhibitory processes would respond under conditions of constant demand over an extended period of time on the task. In a dual approach to this question, we examined how the functional dynamics of this difficult task changed (1), over the course of the entire task session-by comparing functional activations during early blocks of task performance with activations late in task performance, in which time participants had repeated experience with the task, but encountered novel stimuli on each block, and (2) over the course of a task block - by comparing activations during the first half of a task block with those during the second half, in which time participants had repeated experience with the same set of stimuli. This represents a considerable advantage of the WMI paradigm over a standard GO/NOGO paradigm, in which, typically, the same stimuli are repeatedly encountered for the entire duration of task performance. The WMI task allowed us to change the identity of the task stimuli on each block, thus enabling the separation of the effects of item repetition and practice of general cognitive processes. That is, using the WMI task, we were able to assess the effects of brief practice on tonic and phasic activations underlying general WM and inhibitory processes subserving task performance, in addition to item-specific practice effects on both tonic and phasic activations occurring as a result of repeated exposure to the same stimuli over a single block of trials.

Poldrack and his colleagues have investigated similar skill acquisition and item-specific effects in studies examining the neural basis of mirror reading (Poldrack and Gabrieli, 2001; Poldrack et al., 1998). They observed that the acquisition of mirrorreading skill was associated with a reorganization of functional activations, attributed to the fact that the development of the mirror-reading skill was accompanied by a change in the cognitive strategy underlying task performance - a transition from effortful spatial transformation of mirror-reversed letters to easier mnemonic retrieval of stored representations of mirror-reversed letters. On the other hand, repeated stimulus presentations, equivalent to priming, were associated with reduced activation in several areas, including those areas that had shown increases as a result of skill acquisition. That is, priming and skill-learning effects occurred in the same brain regions, even though the loci of activation changed from early to late in performance as a result of skill learning, leading the 
authors to suggest that item-specific learning effects and skilllearning effects share common neural substrates (Poldrack and Gabrieli, 2001).

Guided by these studies, and based on a review of the literature on practice effects (Kelly and Garavan, 2005), we predicted decreases in the WM network as a result of both general task practice and item-specific effects occurring over a single block of trials. Similarly, we predicted that event-related inhibitory activation would decrease over the course of a single block of trials, due to the effects of item repetition. Predictions for an effect of task practice on inhibition-related activations, however, were not as straightforward. Following Hester el al. (2004b), if task practice reduces WM demands and increases neural efficiency in the WM network, then activation should decrease in those regions common to both the WM and inhibitory demands of the task and in those regions uniquely activated by the inhibitory demands of the task, as these regions had shown increased activation in response to increased WM demands. Alternatively, as more attentional resources become available to devote to inhibitory control, activations uniquely related to the inhibitory demands of the task might show further increases. We were also aware that the experimental design allowed us to assess the effects of brief practice on this complex and demanding task, and thus only the earliest and most rapidly occurring effects of practice.

As mentioned above, in addition to individual differences in WM capacity, the ability to control attention has been related to individual differences in performance across a wide range of tasks that place a demand on cognitive control, with recourse to the concept of general fluid intelligence ( $\mathrm{gF}$, Kane and Engle, 2002; see also Duncan, 1995; Duncan et al., 2000). On a neural level, this is reflected in individual differences in the ability to modulate neural activations under conditions demanding high levels of cognitive control (e.g., Kane and Engle, 2002; Mecklinger et al., 2003; Bunge et al., 2001). Thus, in the present study, we were also interested in whether individual differences in the ability to maintain inhibitory control under conditions of high WM demand would be reflected in functional activations, and whether this would interact with practice. If the ability to control attention is amenable to practice, then, because attentional control is a fundamental ability purported to underlie performance across a wide range of cognitively demanding tasks, practice-related improvements in one task might translate to improvements on other, similarly demanding tasks, and even to an improvement of $\mathrm{gF}$.

In order to dissociate tonic WM processes from phasic inhibitory activation to NOGO stimuli, a mixed block- and event-related design was employed, enabling us to identify and dissociate sustained from transient neural activation (Visscher et al., 2003). Thus, in this novel study, we examine individual differences and practice effects in a complex cognitive task which enabled the separation of tonic working memory processes and transient inhibitory processes, in addition to the separation of itemspecific repetition effects and the effects of practice on general task processes.

To facilitate the reader's understanding of our findings, the following points provide a preliminary overview and summary of our findings:

1. Substantial changes in the functional anatomy of task performance occurred in the absence of behavioral changes.
2. Distinct practice-related responses occurred in areas underlying tonic working memory and phasic inhibitory processes as a result of item repetition and general task practice.

3. Both practice and individual differences in task performance were associated with the ability to modulate and maintain activations in frontostriatal areas mediating attentional control.

4. The areas that underlie individual differences in performance can be modulated by practice so that activations become more similar to those shown by better performers.

\section{Methods}

\section{Subjects}

18 right-handed participants (11 female, mean age 28 years) reporting no history of neurological or psychological impairment were recruited from the volunteer pool of the Nathan Kline Institute, Orangeburg, NY. All participants provided informed and written consent prior to taking part in the study.

\section{Task design}

Participants performed the 'Working Memory Inhibitory Task' (WMI task, Hester et al., 2004b), which comprises a Sternberg verbal WM task embedded in a GO/NOGO task. The task was programmed and displayed using E-prime 1.1 (Psychology Software Tools).

In the present study, participants performed the WMI task at the highest level of load used in the Hester et al. (2004b) study, at what is thought to be the capacity limit of human WM storage (Cowan, 2001). Participants were first shown a target memory list of 5 uppercase letters, presented in white font on a black background. This memory list was presented for $6 \mathrm{~s}$ (encoding), followed by a rehearsal period of $8 \mathrm{~s}$ during which time a central fixation crosshair was presented. A series of 120 decision trials then followed, each lasting $1500 \mathrm{~ms}$, after which there was a 32-s concluding rest interval (rest). Each decision trial consisted of a single uppercase letter presented centrally in white font on a black background for $1000 \mathrm{~ms}$, followed by a blank screen for $500 \mathrm{~ms}$. Participants were instructed to indicate as quickly as possible whether each letter was or was not part of the memory list. Participants were trained to use their index finger to press a button for each trial featuring a letter that was not part of the memory list (GO trials) and withhold their response for items that were part of the memory list (NOGO trials) (see Hester et al., 2004b, Fig. 1, for a graphical depiction of the WMI task). Letter stimuli were composed of the 21 consonants of the alphabet. On average, each letter appeared as a target twice, and as a distractor four times, out of the total of 8 blocks. No letter appeared as both a target and a distractor on subsequent trial blocks.

Each block of 120 trials comprised 106 GO and 14 NOGO trials, which were pseudorandomly placed throughout the block in order to create a prepotent response for GO trials. In order to examine short-term practice effects, each participant completed 8 task blocks during one fMRI session. At the conclusion of every second block, a longer (unscanned) rest period was provided. There was a novel and unique target memory set for each block, and block order was identical for each participant. Participants completed the entire 8 experimental blocks in approximately $30 \mathrm{~min}$. 


\begin{tabular}{|c|c|c|c|c|c|c|}
\hline & \multicolumn{3}{|c|}{ EARLY } & \multicolumn{3}{|c|}{ LATE } \\
\hline & $\begin{array}{l}\text { Memory } \\
\text { Set } 1\end{array}$ & & $\begin{array}{l}\text { Memory } \\
\text { Set } 4\end{array}$ & $\begin{array}{l}\text { Memory } \\
\text { Set } 5\end{array}$ & $\cdots$ & $\begin{array}{l}\text { Memory } \\
\text { Set } 8\end{array}$ \\
\hline$\frac{\bar{c}}{\frac{1}{\alpha}}$ & $\begin{array}{c}\text { Probe } 1 \\
\text { Probe } 2 \\
\text { Probe } 3 \\
\ldots \\
\ldots \\
\ldots \\
\text { Probe 60 }\end{array}$ & $\begin{array}{l}\cdots \\
\cdots \\
\cdots\end{array}$ & $\begin{array}{c}\text { Probe } 1 \\
\text { Probe 2 } \\
\text { Probe } 3 \\
\ldots \\
\ldots \\
\ldots \\
\text { Probe } 60\end{array}$ & $\begin{array}{c}\text { Probe } 1 \\
\text { Probe 2 } \\
\text { Probe } 3 \\
\ldots \\
\ldots \\
\ldots \\
\text { Probe } 60\end{array}$ & $\begin{array}{l}\cdots \\
\cdots \\
\cdots\end{array}$ & $\begin{array}{c}\text { Probe } 1 \\
\text { Probe 2 } \\
\text { Probe } 3 \\
\ldots \\
\ldots \\
\ldots \\
\text { Probe } 60\end{array}$ \\
\hline$\frac{N}{\frac{1}{\alpha}}$ & $\begin{array}{c}\text { Probe } 61 \\
\text { Probe } 62 \\
\text { Probe 63 } \\
\ldots \\
\ldots \\
\ldots \\
\text { Probe } 120\end{array}$ & $\begin{array}{l}\cdots \\
\cdots \\
\cdots\end{array}$ & $\begin{array}{c}\text { Probe } 61 \\
\text { Probe } 62 \\
\text { Probe } 63 \\
\ldots \\
\ldots \\
\ldots \\
\text { Probe } 120\end{array}$ & $\begin{array}{c}\text { Probe } 61 \\
\text { Probe } 62 \\
\text { Probe } 63 \\
\ldots \\
\ldots \\
\ldots \\
\text { Probe } 120\end{array}$ & $\begin{array}{l}\cdots \\
\cdots \\
\cdots\end{array}$ & $\begin{array}{c}\text { Probe 61 } \\
\text { Probe } 62 \\
\text { Probe } 63 \\
\ldots \\
\ldots \\
\ldots \\
\text { Probe } 120\end{array}$ \\
\hline
\end{tabular}

Fig. 1. Task conditions. Four time-based task conditions were created on the basis of the combination of two time-based factors, Early/Late and Trials 1/ Trials 2 .

\section{Scanning parameters}

Scanning was conducted on a 1.5 T Siemens VISION scanner in which foam padding was used to restrict head movements. During performance of the task, functional data were collected using contiguous 5-mm axial slices covering the entire brain during a single-shot, $\mathrm{T} 2 *$-weighted echo planar imaging $(\mathrm{EPI})$ sequence $(\mathrm{TE}=$ $50 \mathrm{~ms} ; \mathrm{TR}=2000 \mathrm{~ms} ; \mathrm{FOV}=256 \mathrm{~mm} ; 64 \times 64 \mathrm{~mm}$ matrix size inplane resolution). High-resolution T1-weighted structural magnetization prepared gradient echo (MPRAGE) images (FOV $=307 \mathrm{~mm}$, isotropic $1.2 \mathrm{~mm}$ voxels) were acquired to allow subsequent activation localization and spatial normalization. Visual stimuli were delivered using an IFIS-SA stimulus-delivery system (MRI Devices Corp., Waukesha, Wisconsin), which was equipped with a head-coil-mounted $640 \times 480 \mathrm{LCD}$ panel. This shielded LCD screen is mounted on the head-coil, directly in the subjects' line of vision.

\section{Image analysis}

All data processing was conducted using the AFNI software package (http://afni.nimh.nih.gov/afni/). Following image reconstruction, the time-series data were time-shifted using Fourier interpolation to correct for differences in slice acquisition times. Each of the four scanning runs was motion corrected by volume registering each $3 \mathrm{D}$ image to a single "base" image (the middle image of the time-series), using Fourier interpolation to perform least squares alignments of three translational and three rotational parameters. The time-series from each run were then concatenated into one dataset and reregistered to the base image of the first run to correct for participant motion between scanning runs. Voxels lying outside the brain were then removed.

\section{Comparisons of interest}

To examine the effects of short-term practice on the task, four time-based conditions were created on the basis of two time-based comparison factors. The first of these factors was an EARLY-LATE comparison: the experiment was separated into an EARLY (first 4 blocks) period and a LATE (last 4 blocks) period. The second time factor aimed to examine activation over the course of a single experimental block of trials and was called the TRIALS 1-2 factor: each block of 120 trials was split in two-TRIALS 1 (the first 60 trials) and TRIALS 2 (the second 60 trials). This factorial design gave rise to 4 time-based conditions (see Fig. 1): Early Trials 1, Early Trials 2, Late Trials 1, Late Trials 2.

A mixed regression analysis was carried out which included event-related regressors coding for correct inhibitions and errors of commission, block regressors corresponding to the encoding and rehearsal periods, and four square-wave regressors corresponding to ongoing trial activity in each time condition. In this regression analysis, tonic activation related to the ongoing WM processes was calculated as a percentage change relative to the resting baseline.

A second, event-related (ER) analysis estimated activation separately for successful inhibitions (STOPS) for each of the four time conditions and for errors of commission collapsed across all conditions (necessary due to the small number of errors). A deconvolution analysis calculated separate impulse response functions (IRFs) at 2-s resolution for STOPS in each of the conditions and for errors. Although the stimulus stream was presented at $1.5 \mathrm{~Hz}$, all events of interest were time locked to the beginning of the 2-s whole brain volume acquisition during presentation of the task. A nonlinear regression program then determined the best-fitting gamma-variate function for these IRFs as described previously (Garavan et al., 1999). Event-related activation for each condition was then calculated by expressing the area under the gamma-variate curve as a percentage of the area under the baseline, the baseline in this analysis being the ongoing working memory trial period activity. The rest period was censored from this analysis and nuisance regressors coding for the encoding and rehearsal periods were included in order to remove as much of the variance associated with these periods as possible.

The percentage change (tonic activation) and percentage area (event-related activation) maps were resampled at $1-\mathrm{mm}^{3}$ resolution and warped into standard Talairach space (Talairach and Tournoux, 1988), then spatially blurred with a 3-mm isotropic rms Gaussian kernel. Group activation maps for each condition were determined with one-sample $t$ tests against the null hypothesis of zero activation changes relative to baseline. Significant voxels passed a voxelwise statistical threshold $(t=3.965, P=0.001)$ and were required to be part of a larger cluster of contiguous significant voxels $(140 \mu \mathrm{l}$ in the block analysis and $132 \mu \mathrm{l}$ in the event-related analysis). This cluster size was determined though Monte Carlo simulations based on the number of active voxels in each of the $t$ test maps and resulted in a $<5 \%$ probability of a cluster surviving due to chance.

In both the block- and event-related analyses, the separate activation maps for each condition were combined to produce an OR map. An OR map includes the voxels of activation that are significant in any one of the constituent condition maps. In addition, to identify regions of activation common to both tonic working memory processes and phasic inhibition, an AND map was created which included voxels of activation common to the block (tonic) and event-related (STOPS) activation maps. The mean activation for clusters of significant voxels in these combined maps was calculated for the purposes of a functionally defined ROI analysis. These activation data were then entered into a $2 \times 2$ $($ EARLY-LATE $\times$ TRIALS $1-2)$ factorial repeated measures 
ANOVA, corrected using a modified Bonferroni procedure for multiple comparisons (Keppel, 1991). Behavioral data were analyzed using the same $2 \times 2$ factorial repeated measures ANOVA.

\section{Results}

\section{Behavioral performance}

Fig. 2 illustrates behavioral performance across the four time conditions of the task. There were no behavioral effects of brief practice on the task. In a series of $2 \times 2$ repeated measures ANOVAs on reaction time (RT), accuracy (correct inhibitions) and errors of omission data, no main effects for either the EARLYLATE factor, nor the TRIALS 1-2 factor were was observed $(F(1,17)<1, P>0.05$ in all cases $)$. To confirm the absence of a behavioral effect of practice on accuracy and RT, we compared an average Early score to an average Late score, and average Trials 1 and Trials 2 scores in a series of paired-samples $t$ tests. In all cases $P>0.05$, confirming the null result.

There were no interactions for RT or accuracy. An interaction for errors of omission revealed that while omissions decreased from the first $(6 \%)$ to second half $(4.3 \%)$ of the EARLY blocks, they increased from the first $(4.9 \%)$ to the second half $(7 \%)$ of the LATE blocks. Only the latter effect was significant $t(1,17)=1.258$, $P<0.05$.

\section{Tonic working memory activations}

The mixed functional analysis revealed a largely left-hemisphere based network comprising cortical and subcortical areas underlying tonic WM processes involved in trial-to-trial task performance (see Table 1). Table 1 also indicates which areas showed a significant main effect of either the Early-Late factor or the Trials $1-2$ factor in a $2 \times 2$ ANOVA conducted on the tonic activation data.

\section{Early-Late comparison}

There were significant decreases in activation across a large prefrontal and subcortical network from early to late in task performance - that is, over the duration of the entire task session. These decreases, indicated by a significant main effect of the EarlyLate comparison, occurred bilaterally in large clusters comprising lateral PFC, insula, striatum, and thalamus as well as in the presupplementary motor area (pre-SMA) and dorsal tegmental brainstem area (see the BLUE areas of Fig. 3). Only the decrease in activation observed in the large right-hemisphere frontostriatal cluster failed to reach the statistical threshold adjusted for multiple ROI comparisons, all other activation changes were significant at this stricter threshold $(P<0.04)$.

\section{Trials 1-2 comparison}

In contrast to the frontostriatal decreases observed in the EarlyLate comparison, the main effect for the Trials 1-2 factor revealed that, over the course of a block of trials, tonic WM activation decreased in left posterior parietal cortex and areas of cingulate cortex - the left ACC and a more posterior cingulate area including the corpus callosum. These changes were less robust however, and only the decrease in activation in the posterior cingulate/callosal area remained significant after correction for multiple comparisons. Two areas of decreased deactivation were also significant at this stricter threshold-in right somatosensory cortex and a region of the corpus callosum (splenium).

Only one cluster, in the right inferior frontal gyrus (IFG), showed a significant interaction, which indicated that while activation during the early part of task performance decreased over the course of a single block of trials, during later task performance, it increased over the course a block.

Some areas showed no change in activation either over the course of a block of trials, or over the entire 8 blocks. These included left premotor, right SMA, and left motor areas. Similarly, a left frontopolar area was unaffected by task practice, as were areas of left inferior parietal and supramarginal cortex, the right cerebellum and left caudate.

It is possible that fatigue, rather than practice, can account for the decreases in activation observed. In order to differentiate between these two explanations, we carried out an additional analysis in which we selected the 5 participants who showed the greatest disimprovement from early to late in task performance

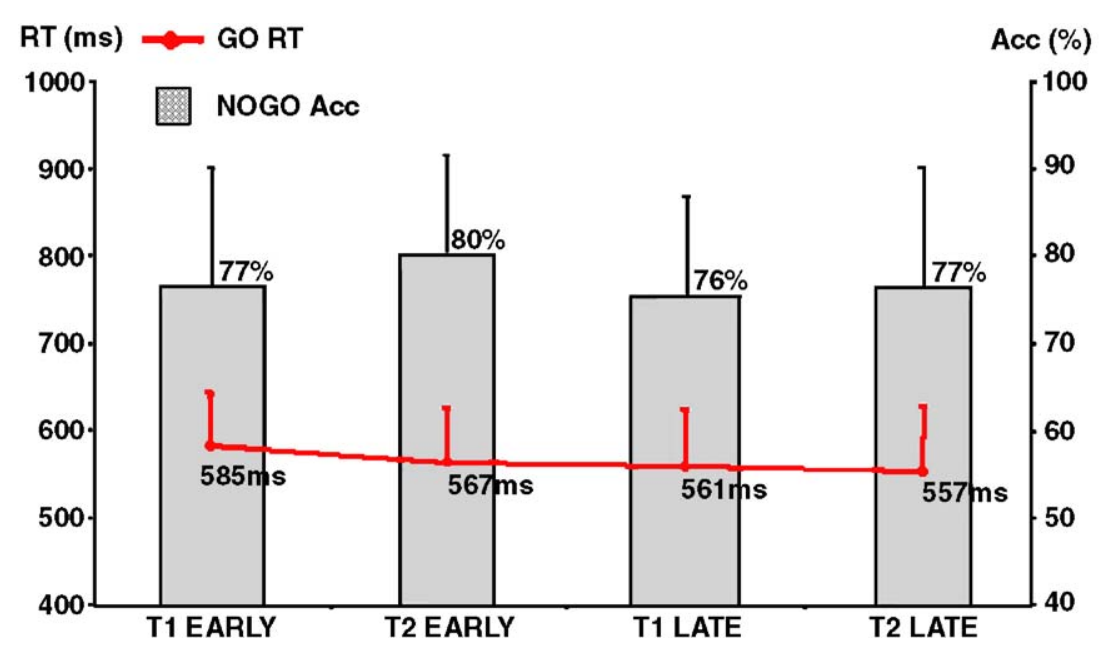

Fig. 2. Behavioral performance across the four time conditions. There were no effects of practice on either response times (RT), presented in milliseconds (ms) or NOGO accuracy (Acc) presented as a percentage of the total number of NOGO stimuli. 
Table 1

Functionally defined regions tonically activated during trial-to-trial working memory performance

\begin{tabular}{|c|c|c|c|c|c|c|c|c|}
\hline \multirow[t]{2}{*}{ Structure } & \multirow[t]{2}{*}{$\mathrm{BA}$} & \multirow[t]{2}{*}{ HS } & \multirow[t]{2}{*}{ Vol $(\mu \mathrm{l})$} & \multicolumn{3}{|c|}{ Center of mass } & \multicolumn{2}{|l|}{ Activation change } \\
\hline & & & & $x$ & $y$ & $z$ & EARLY-LATE & TRIALS 1-2 \\
\hline & & & & & & & $\begin{array}{l}\downarrow \text { Activation decreases } \\
\uparrow \text { Activation increases }\end{array}$ & \\
\hline \multicolumn{9}{|l|}{ Frontal lobe } \\
\hline \multirow{2}{*}{$\begin{array}{l}\text { Large cluster comprising middle/inferior frontal } \\
\text { Gyri, insula, striatum, thalamus }\end{array}$} & $9 / 13$ & $\mathrm{R}$ & 4013 & 27.6 & 8.3 & 9.8 & $\downarrow$ & \\
\hline & $\begin{array}{l}9 / 44 / 45 / \\
46 / 47 / 13\end{array}$ & $\mathrm{~L}$ & 15,554 & -32.3 & 9.5 & 11.7 & $\downarrow$ & \\
\hline Middle frontal & 6 & $\mathrm{~L}$ & 622 & -37.3 & -2.6 & 45.9 & & \\
\hline Inferior frontal & 9 & $\mathrm{R}$ & 407 & 36.1 & 8.6 & 26.6 & Interaction $\mathrm{E} 1-2 \downarrow \mathrm{L} 1-2 \uparrow$ & \\
\hline Superior/Middle frontal & 8 & $\mathrm{R}$ & 328 & 25.3 & 23.0 & 44.3 & Increased deactivation & \\
\hline Medial frontopolar & 10 & $\mathrm{~L}$ & 168 & -0.5 & 15.0 & 48.9 & & \\
\hline BL medial frontal - PreSMA & 8 & BL & 187 & -0.5 & 15.0 & 48.9 & $\downarrow$ & \\
\hline Medial frontal/cingulate & $8 / 32$ & $\mathrm{R}$ & 727 & 9.7 & 14.9 & 38.0 & & \\
\hline Anterior cingulate & $24 / 32$ & $\mathrm{~L}$ & 152 & -7.8 & 14.1 & 38.8 & & $\downarrow$ \\
\hline \multicolumn{9}{|l|}{ Parietal lobe } \\
\hline $\mathrm{R}$ postcentral $\mathrm{BA} 1 / 3$ & $1 / 3$ & $\mathrm{R}$ & 156 & 63.0 & -16.2 & 32.2 & & $\begin{array}{l}\text { Decreased } \\
\text { deactivation }\end{array}$ \\
\hline Pre/Post central & $4 / 1$ & $\mathrm{~L}$ & 2203 & -41.3 & -22.6 & 48.1 & & \\
\hline Postcentral BA 5 & 5 & $\mathrm{~L}$ & 215 & -38.1 & -40.4 & 61.6 & & \\
\hline Inferior parietal & 40 & $\mathrm{~L}$ & 408 & -35.8 & -40.8 & 40.3 & & \\
\hline Precuneus & 7 & $\mathrm{~L}$ & 779 & -25.1 & -60.1 & 36.5 & & $\downarrow$ \\
\hline Precunues/Supramarginal gyrus & $7 / 40$ & $\mathrm{~L}$ & 201 & -28.8 & -47.8 & 32.2 & & \\
\hline Cingulate/Corpus callosum & & $\mathrm{BL}$ & 3350 & 1.3 & -25.9 & 21.4 & & $\downarrow$ \\
\hline Corpus callosum - splenium & & BL & 267 & -1.3 & -41.6 & 8.8 & & $\begin{array}{l}\text { Decreased } \\
\text { deactivation }\end{array}$ \\
\hline \multicolumn{9}{|l|}{ Subcortical } \\
\hline Brainstem - dorsal tegmental & & $\mathrm{R}$ & 190 & 11.7 & -25.5 & -13.3 & $\downarrow$ & \\
\hline Brainstem - dorsal tegmental/parahippocampus & & $\mathrm{L}$ & 609 & -10.6 & -28.9 & -11.1 & $\downarrow$ & \\
\hline Caudate & & $\mathrm{L}$ & 65 & -3.1 & 5.5 & 17.1 & & \\
\hline Cerebellum - culmen & & $\mathrm{R}$ & 699 & 9.3 & -47.8 & -11.0 & & \\
\hline
\end{tabular}

Areas showing activation changes as a result of the time manipulation are indicated in the rightmost columns.

Positive values for $x, y$, and $z$ coordinates denote locations right, anterior, and superior relative to the anterior commissure. BA-Brodmann's area, HScerebral hemisphere.

Regions which showed practice-related changes in activation changes are shaded in grey, unshaded areas showed no significant effects of practice.

(i.e., when performance over the first four blocks was compared to that of the last four). These participants' accuracy decreased by an average of $13.7 \%$ from early to late in performance, a change which may reflect the effects of increasing fatigue. Three out of these five participants were also among a group of five participants identified as showing the greatest increases in errors of omission, consistent with these participants suffering performance decrements as a result of greater fatigue. If the decreases in activation that were observed are a result of increased fatigue, then the 'disimprovers' group should show greater decreases in activation. In contrast, when we examined the percent activation change from early to late in those regions which showed a practice-related decrease, the 'disimprovers' group showed little or no change in activation in those areas.

\section{Event-related inhibitions (STOPS)}

The event-related analysis revealed a widespread, largely rightlateralized, network of activation including lateral and medial frontal areas, parietal cortex, and subcortical areas (see Table 2). Table 2 presents the areas showing a significant main effect of either the Early-Late factor or the Trials $1-2$ factor in a $2 \times 2$ ANOVA conducted on the STOPS activation data.

\section{Early-Late comparison}

In contrast to the decreases observed in tonic WM-related activity, main effects for the Early-Late comparison in a $2 \times 2$ ANOVA conducted on the event-related inhibitions data indicated that bilateral PFC, right primary motor cortex, and bilateral inferior parietal cortex showed increased activation for STOPS in the later four blocks relative to the earlier four blocks of task performance (see the red areas of Fig. 4A). At the stricter statistical threshold adjusted for multiple ROI comparisons $(P<0.02)$, increases in right premotor, dIPFC and bilateral inferior parietal/supramarginal gyri remained significant. The Early-Late comparison also revealed four areas showing decreased activation for STOPS - the right putamen, right lateral premotor area, left medial BA6 (SMA), and left occipital gyrus (see the BLUE areas of Fig. 4A). Only the decrease in activation occurring in the right putamen survived the stricter, corrected threshold.

The practice-related changes in activation for STOPS occurred in the absence of tonic activation (baseline) changes in these areas, with the exception of an area of right superior PFC (BA 8), which showed increased activation from early to late in task performance, and also showed increases in tonic activation from early to late in performance. 


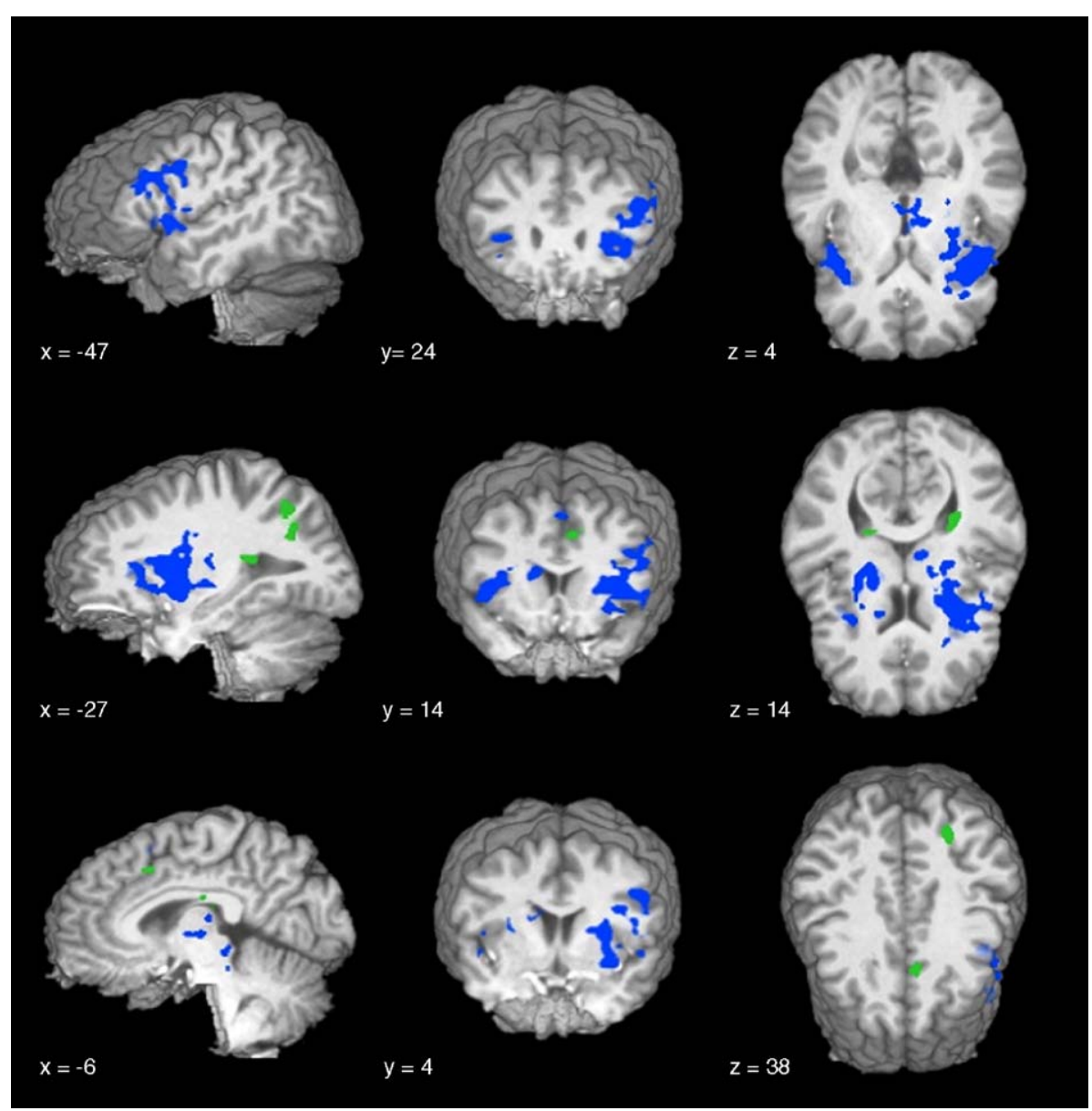

Fig. 3. Areas showing decreases in tonic working memory-related activations for the Early-Late comparison (blue) and for the Trials $1-2$ comparison (green).

\section{Trials 1-2 comparison}

Decreased event-related activation for STOPS over the course of a block of trials was observed in a largely prefrontal and subcortical network of areas, the parietal cortex and cerebellum (see the blue areas in Fig. 4B). On the other hand, increased activation for STOPS over the course of a block of trials was observed in posterior cortex - in right primary motor cortex, left superior temporal/supramarginal cortex, right superior parietal cortex, and areas of the occipital cortex (see the red areas in Fig. 4B).

As was the case in the Trials 1-2 comparison for tonic activations, these effects were less robust than those in the EarlyLate comparison. Decreases in activation which survived the multiple ROI corrected threshold $(P<0.02)$ were observed in the left hemisphere: in inferior frontal/insular cortex and putamen, cerebellum, and inferior parietal cortex. Increased activation surviving the stricter threshold was observed in right primary motor and superior parietal cortices and bilaterally in occipital cortex. Several of these areas showed a concomitant change in baseline tonic activation-both left superior parietal and lingual gyri showed decreased deactivations tonically, and primary motor cortex showed an increase in tonic activation.

A number of areas active for STOPS showed significant interactions. These were right superior and medial frontal gyri, and inferior parietal and occipital cortices. Typically, these interactions reflected a decrease in activation over the course of a block of trials during the early period of performance but increased activation over the course of a block during later performance-a U-shaped function. An exception to this pattern was the superior frontal area which showed an increase over the earlier trials and a decrease for trials later in performance.

While practice-related changes in activation occurred in the majority of areas active for STOPS, there were a number of areas which showed no change in activation: right dlPFC, bilateral anterior PFC, left fusiform cortex, and bilateral areas of parietal cortex.

In order to examine whether increasing interference could explain the observed increases in activation, an additional analysis compared the increases in activation for the group of five participants who showed the greatest slowing from early to late in performance (an average increase in RT of $45.7 \mathrm{~ms}$ ) and a group of five participants who showed the greatest decrease in RT from early to late in performance (an average decrease in RT of $79.6 \mathrm{~ms}$ ). If the increases in activation are due to increased interference arising from the same stimuli being used as both targets and distractors, then the group whose response times increased, who presumably experienced greater increases in interference, should show a greater increase in activation from early to late in performance. We compared the change in event-related activation in those regions showing early-late activation increases between the 'slow and 'fast' groups. There were no significant differences in the increases in activation for these two groups $(P>0.05$ in all comparisons). 
Table 2

Regions of event-related activation during successful response inhibitions (STOPS)

\begin{tabular}{|c|c|c|c|c|c|c|c|c|c|}
\hline \multirow[t]{2}{*}{ Structure } & \multirow[t]{2}{*}{ BA } & \multirow[t]{2}{*}{ HS } & \multirow[t]{2}{*}{$\operatorname{Vol}(\mu \mathrm{l})$} & \multicolumn{3}{|c|}{ Center of mass } & \multicolumn{2}{|l|}{ Activation change } & \multirow{2}{*}{$\begin{array}{l}\text { Performance } \\
\text { effect? }\end{array}$} \\
\hline & & & & $x$ & $y$ & $z$ & EARLY-LATE & TRIALS 1-2 & \\
\hline & & & & & & & $\begin{array}{l}\downarrow \text { Activation decreases } \\
\uparrow \text { Activation increases }\end{array}$ & & \\
\hline \multicolumn{10}{|l|}{ Frontal lobe } \\
\hline \multirow[t]{6}{*}{ Middle frontal } & 6 & $\mathrm{R}$ & 582 & -24.8 & -7.3 & 53.3 & $\downarrow$ & & \\
\hline & $10 / 46$ & $\mathrm{R}$ & 375 & -31.1 & 31.4 & 20.5 & & & \\
\hline & 9 & $\mathrm{R}$ & 332 & 38.8 & 33.4 & 27.1 & $\uparrow$ & & \\
\hline & 9 & $\mathrm{R}$ & 262 & -50.1 & 15.0 & 33.0 & $\uparrow$ & $\downarrow$ & \\
\hline & 10 & $\mathrm{R}$ & 226 & -34.8 & 45.1 & 21.2 & & $\downarrow$ & \\
\hline & 10 & $\mathrm{~L}$ & 178 & 32.4 & 42.3 & 4.6 & & & \\
\hline \multirow[t]{2}{*}{ Inferior frontal/insula/putamen } & $47 / 13$ & $\mathrm{R}$ & 2573 & -26.7 & 11.8 & -3.2 & & $\downarrow$ & $*$ \\
\hline & $47 / 13$ & $\mathrm{~L}$ & 1552 & 27.5 & 12.5 & -0.6 & & $\downarrow$ & $*$ \\
\hline Inferior frontal/precentral & 44 & $\mathrm{R}$ & 344 & -53.0 & 9.0 & 10.7 & $\uparrow$ & $\downarrow$ & $*$ \\
\hline \multirow{3}{*}{ Inferior frontal } & $9 / 45 / 46$ & $\mathrm{R}$ & 580 & -48.7 & 15.7 & -0.6 & $\uparrow$ & & * \\
\hline & 9 & $\mathrm{R}$ & 157 & -42.3 & 6.5 & 25.8 & $\uparrow$ & & \\
\hline & 9 & $\mathrm{~L}$ & 516 & 43.9 & 3.6 & 27.7 & $\uparrow$ & & \\
\hline \multirow[t]{3}{*}{ Superior frontal } & 8 & $\mathrm{R}$ & 353 & -21.7 & 16.2 & 33.5 & $\uparrow$ & & \\
\hline & & & & & & & Interaction $\mathrm{E} 1-2 \uparrow \mathrm{L} 1-2 \downarrow$ & & \\
\hline & 6 & $\mathrm{R}$ & 236 & -20.8 & 10.2 & 56.6 & & & \\
\hline \multirow[t]{5}{*}{ Medial frontal } & $6 / 24$ & $\mathrm{R}$ & 151 & -5.4 & -12.3 & 53.6 & & $\downarrow$ & \\
\hline & & & & & & & Interaction $\mathrm{E} 1-2 \downarrow \mathrm{L} 1-2 \uparrow$ & & \\
\hline & 6 & $\mathrm{R}$ & 378 & -3.0 & -0.9 & 55.6 & & $\downarrow$ & \\
\hline & $6 / 8$ & BL & 188 & -2.7 & 15.1 & 47.1 & & $\downarrow$ & \\
\hline & $6 / 24$ & $\mathrm{~L}$ & 183 & 10.2 & -2.8 & 50.4 & $\downarrow$ & $\downarrow$ & \\
\hline \multicolumn{10}{|l|}{ Temporal lobe } \\
\hline Superior temporal/supramarginal & 40 & $\mathrm{~L}$ & 156 & 55.3 & -54.3 & 20.4 & $\uparrow$ & $\uparrow$ & \\
\hline Fusiform & 37 & $\mathrm{~L}$ & 147 & 42.4 & -57.2 & -12.0 & & & \\
\hline \multicolumn{10}{|l|}{ Parietal lobe } \\
\hline \multirow[t]{4}{*}{ Inferior parietal } & 40 & $\mathrm{R}$ & 6192 & -29.7 & -55.7 & 41.3 & Interaction $\mathrm{E} 1-2 \downarrow \mathrm{L} 1-2 \uparrow$ & & \\
\hline & 40 & $\mathrm{R}$ & 203 & -44.4 & -31.3 & 31.6 & & $\downarrow$ & \\
\hline & 40 & $\mathrm{~L}$ & 478 & -51.4 & -34.9 & 48.2 & & & \\
\hline & 40 & $\mathrm{~L}$ & 214 & 44.2 & -40.6 & 50.0 & & & \\
\hline \multirow[t]{2}{*}{ Inferior parietal/supramarginal } & 40 & $\mathrm{R}$ & 133 & -58.6 & -45.0 & 35.5 & $\uparrow$ & & \\
\hline & 40 & $\mathrm{~L}$ & 321 & 53.6 & -43.2 & 31.6 & $\uparrow$ & & \\
\hline \multirow[t]{3}{*}{ SPL/Precuneus } & 7 & $\mathrm{R}$ & 142 & -29.6 & -49.0 & 61.2 & & & \\
\hline & 7 & $\mathrm{R}$ & 134 & -20.0 & -50.7 & 60.4 & & $\uparrow$ & \\
\hline & 7 & $\mathrm{~L}$ & 3046 & 18.6 & -63.0 & 43.7 & & & \\
\hline Postcentral & 4 & $\mathrm{R}$ & 272 & -13.4 & -34.9 & 63.0 & $\uparrow$ & $\uparrow$ & \\
\hline \multicolumn{10}{|l|}{ Occipital lobe } \\
\hline Middle occipital & $18 / 19$ & $\mathrm{R}$ & 275 & -32.7 & -88.7 & 4.9 & Interaction $\mathrm{E} 1-2 \downarrow \mathrm{L} 1-2 \uparrow$ & & \\
\hline Middle occipital/inferior temporal & 37 & $\mathrm{~L}$ & 297 & 37.9 & -64.9 & 2.4 & $\downarrow$ & $\uparrow$ & \\
\hline Lingual & 18 & $\mathrm{~L}$ & 254 & 18.7 & -82.5 & -4.6 & & & \\
\hline Lingual/Cuneus & 18 & $\mathrm{~L}$ & 178 & 14.3 & -69.9 & 5.2 & & $\uparrow$ & \\
\hline \multicolumn{10}{|l|}{ Subcortical } \\
\hline Putamen & & $\mathrm{R}$ & 200 & -17.9 & -3.2 & 11.4 & $\downarrow$ & $\downarrow$ & \\
\hline Caudate & & $\mathrm{R}$ & 251 & -3.7 & 3.3 & 9.2 & & $\downarrow$ & \\
\hline Cerebellum-culmen & & $\mathrm{L}$ & 161 & 28.2 & -60.4 & -26.7 & & $\downarrow$ & \\
\hline
\end{tabular}

Activation changes observed as a result of the time-based comparisons are also shown.

Positive values for $x, y$, and $z$ coordinates denote locations right, anterior and superior relative to the anterior commissure. BA-Brodmann's area, HScerebral hemisphere.

Regions which showed practice-related changes in activation changes are shaded in grey, unshaded areas showed no significant effects of practice.

\section{Individual differences}

In order to examine the relationship between activation, behavioral performance and practice, a split-half comparison was performed by dividing the sample based on the total number of correct inhibitions across the entire 8 blocks of the task, to form high and low performance groups. A main effect of performance in a 2 (group) $\times 2$ (Early-Late) $\times 2$ (Trials $1-2)$ ANOVA carried out on the behavioral and event-related inhibitions data indicated that the high performance group was significantly more accurate on the task 


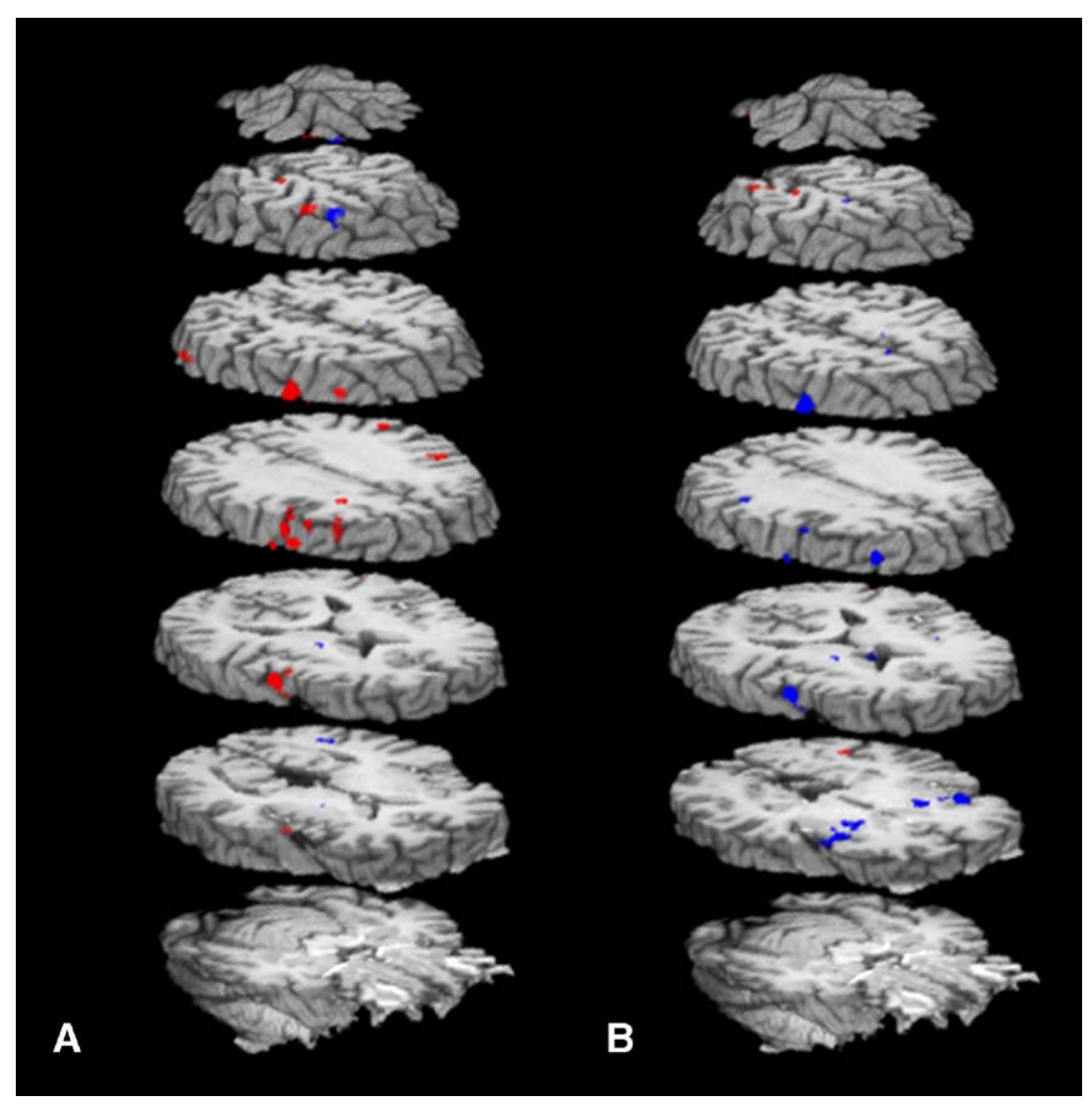

Fig. 4. Regions of event-related activation during successful inhibitions (STOPS) sensitive to practice effects in (A) the Early-Late comparison and (B) the Trials 1-2 comparison. Areas of increase are shown in red, and areas of decrease are shown in blue.

(84\% versus $70 \%, P<0.001)$ and showed significantly more activation for correct inhibitions in two clusters: right dIPFC and a large cluster comprising left inferior frontal/insular cortex and putamen. In contrast, poorer performers showed greater activation in a right inferior parietal/supramarginal area. There were no interaction effects between performance and practice in the behavioral data. An interaction between performance and practice was observed in two brain areas: the right IFG/insular/putamen cluster and right IFG (area 44/35) showed a Trials 1-2 $\times$ Performance interaction such that over the course of a block of trials, poorer performers showed a decrease in activation, but the high performance group showed no change in activation. When the same ANOVA was performed on the tonic functional data, no significant effects of performance on tonic WM activations were observed.

The dissociation between increased inhibitory activation frontally for the high performance group but posteriorly for the low performance group was significant-in two 2 (Area) $\times 2$ (Performance) ANOVAs comparing activation in the right dIPFC and right IFG/insula areas to that of the inferior parietal cortex, there were significant Area $\times$ Performance interactions $(P<0.05$ in both cases, see Fig. 5).

\section{Common regions of activation}

There were several regions of overlap for tonic, WM activations and transient, inhibitory activations (STOPS). These are presented in Table 3 and Fig. 6. A $2 \times 2$ ANOVA revealed main effects of the Early-Late and Trials 1-2 factors for STOPS (event-related) activity only-there were no effects of practice on tonic activations. The
Early-Late comparison revealed increased activation in right inferior frontal cortex/insula late in task performance, in contrast to the right putamen, where activation decreased both across a task block (Trials $1-2$ ) and across all 8 task blocks (Early-Late). Left inferior frontal/ insular cortex also showed a decrease over the course of a task block. There were no effects of performance in these areas.

\section{Error-related activation}

Errors of commission in the WMI task activated a network of areas including dorsolateral and medial prefrontal, anterior cingulate, and insular areas (see Table 4). Due to the relatively small number of errors, analysis was collapsed across all four time conditions, and practice effects were not examined. There were, however, significant performance effects in both right and left dIPFC wherein high performers showed increased activation for errors when compared to poorer performers.

\section{Discussion}

The present study represents a novel examination of brief practice on a GO/NOGO task, and specifically, on inhibitory performance under a dual-task situation of high WM task load. It is also a novel examination of the effects of brief practice of the WMI task on general cognitive task processes and the effects of itemspecific practice occurring as a result of repeated exposure to the same stimuli over a single block of trials. We observed that withholding a prepotent response to items held in WM activated a 


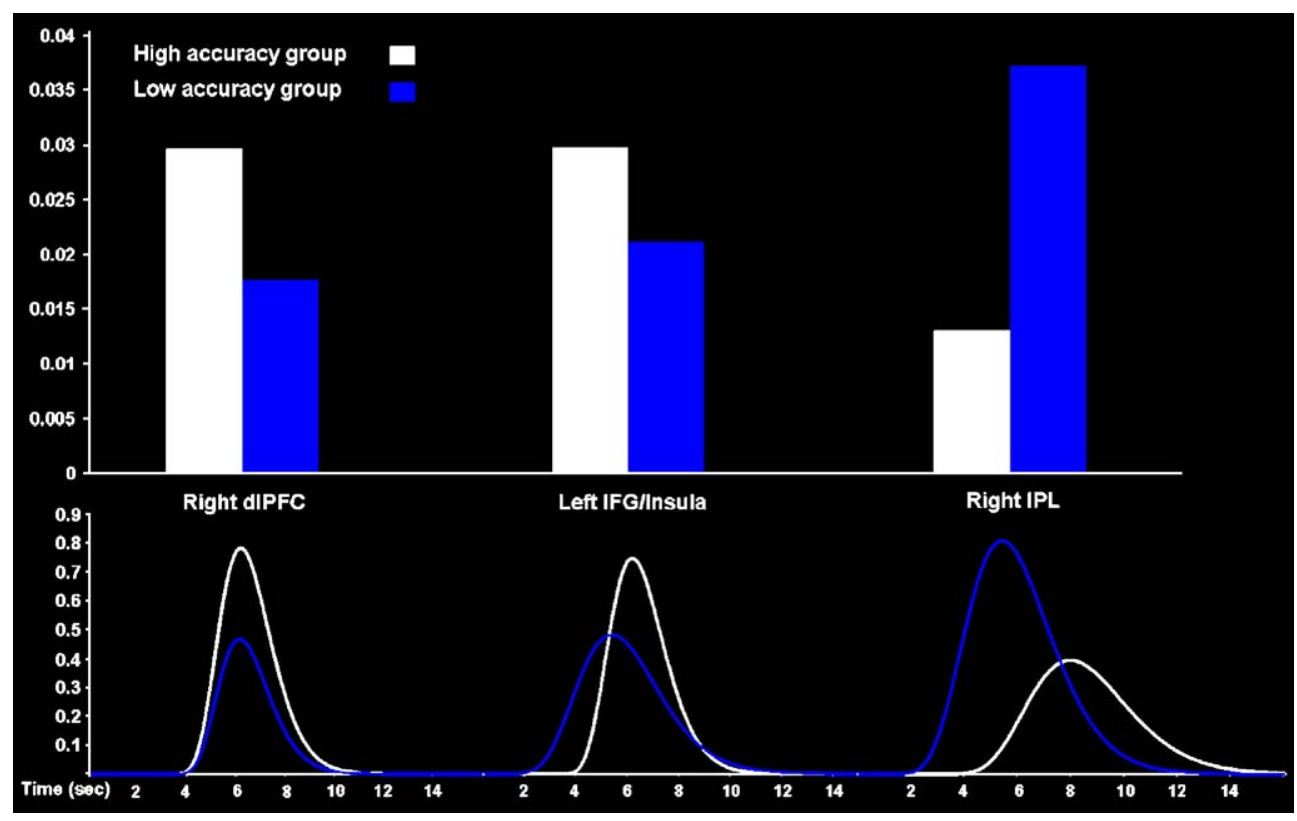

Fig. 5. Area $\times$ Performance dissociation. The high performance group demonstrated greater activation for STOPS in dorsolateral prefrontal and inferior frontal/ insular cortex (extending to the putamen), while poorer performers demonstrated greater activation in inferior parietal cortex. In the top panel, the dissociation is depicted in terms of mean \%area under the curve measures in each area, the bottom panel displays the associated mean gamma-variate curve describing the hemodynamic response function in each area.

predominantly right-hemisphere network of regions, including middle and inferior frontal cortex, insula, midline frontal areas (SMA, pre-SMA, and ACC), inferior parietal cortex, striatum and thalamus, consistent with Hester et al. (2004b) and previous neuroimaging studies of response inhibition (e.g., Garavan et al., 1999; Kelly et al., 2004; Liddle et al., 2001; Rubia et al., 2003; Watanabe et al., 2002). Errors of commission activated lateral frontal, midline, and insular areas also consistent with previous studies (Hester et al., 2004b; Holroyd et al., 2004; Ullsperger and Von Cramon, 2003; Ridderinkhof et al., 2004; Kiehl et al., 2000). In addition, the examination of tonic WM processes revealed activation in a primarily left-hemisphere network of regions, including left dorso- and ventrolateral PFC, insula, medial frontal cortex (pre-
SMA/ACC), bilateral posterior parietal cortices, the striatum, and the cerebellum, in line with previous neuroimaging studies of verbal working memory (e.g., Baddeley, 2003; Jonides et al., 1998a; Manoach et al., 1997, 2003; Smith and Jonides, 1998; Wager and Smith, 2003). Consistent with Hester et al. (2004b), participants were able to maintain successful inhibitory control over prepotent responses, despite high WM load (5 items). This was achieved through the activation of several areas specific to the inhibitory demands of the task, in addition to a network of brain regions commonly activated by both the WM and inhibitory demands of the task, including left dlPFC and parietal cortex, bilateral inferior frontal gyrus (IFG)/insular cortex, and the right putamen.

Table 3

Common regions of activation for phasic STOPS and tonic working memory processes

\begin{tabular}{|c|c|c|c|c|c|c|c|c|}
\hline \multirow[t]{2}{*}{ Structure } & \multirow[t]{2}{*}{ BA } & \multirow[t]{2}{*}{ HS } & \multirow[t]{2}{*}{ Vol $(\mu \mathrm{l})$} & \multicolumn{3}{|c|}{ Center of mass } & \multicolumn{2}{|c|}{ Activation change for STOPS } \\
\hline & & & & $x$ & $y$ & $z$ & EARLY-LATE & TRIALS 1-2 \\
\hline & & & & & & & $\begin{array}{l}\downarrow \text { Activation decreases } \\
\uparrow \text { Activation increases }\end{array}$ & \\
\hline \multicolumn{9}{|l|}{ Frontal lobe } \\
\hline Middle/frontal & 9/46 & $\mathrm{L}$ & 144 & -46.1 & 3.9 & 31.9 & & \\
\hline \multirow[t]{2}{*}{ Inferior frontal/insula } & $47 / 13$ & $\mathrm{R}$ & 137 & 37.6 & 16.9 & 2.1 & $\uparrow$ & \\
\hline & $47 / 13$ & $\mathrm{~L}$ & 442 & -35.0 & 15.5 & 3.2 & & $\downarrow$ \\
\hline \multicolumn{9}{|l|}{ Parietal lobe } \\
\hline Inferior parietal & 40 & $\mathrm{~L}$ & 193 & -35.5 & -40.5 & 39.8 & & \\
\hline Precuneus & 7 & $\mathrm{~L}$ & 503 & -24.8 & -59.9 & 38.4 & & \\
\hline \multicolumn{9}{|l|}{ Subcortical } \\
\hline Putamen & & $\mathrm{R}$ & 127 & 17.6 & -3.7 & 12.7 & $\downarrow$ & $\downarrow$ \\
\hline
\end{tabular}

Positive values for $x, y$, and $z$ coordinates denote locations right, anterior, and superior relative to the anterior commissure. BA-Brodmann's area, HScerebral hemisphere.

Regions which showed practice-related changes in activation changes are shaded in grey, unshaded areas showed no significant effects of practice. 


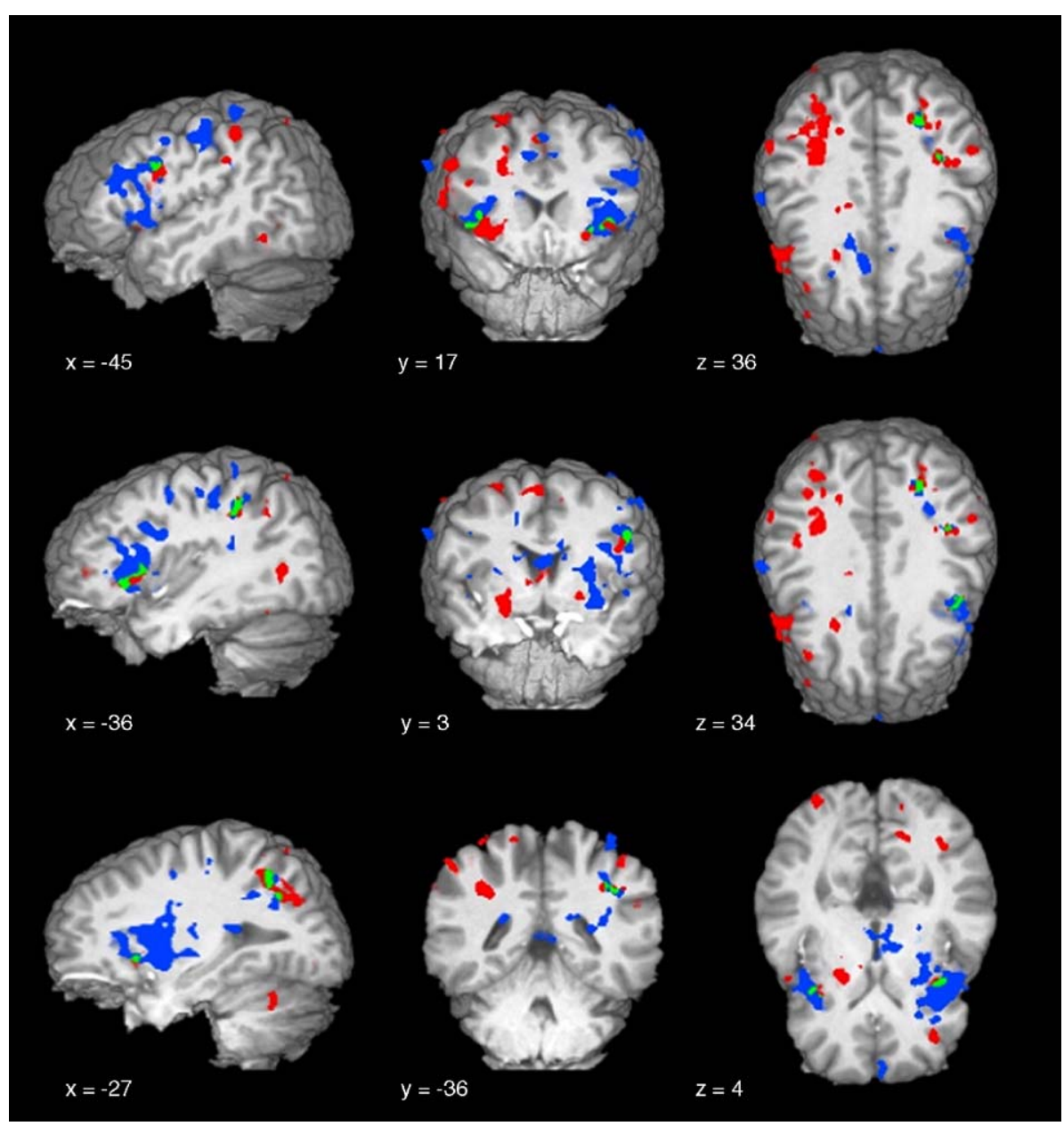

Fig. 6. Overlap of tonic WM-related activations (red) and phasic inhibitory activations (blue). Areas of direct overlap are shown in green.

In the following sections, we highlight and discuss each of the study's major findings in terms of the functional roles of different brain regions, process-specific versus item-specific repetition effects of practice, common and unique cognitive and neural resources, and individual differences, in the context of theories of WM and executive attention. Primarily, we show that:

1. Substantial changes in the functional anatomy of task performance occurred in the absence of behavioral changes.
2. There were distinct practice-related responses in areas underlying tonic working memory and phasic inhibitory processes. For WM activations, both item repetition and task practice lead to activation decreases. In contrast, while phasic inhibitory activations decreased in association with item repetition, inhibitory activations increased as a result of task practice.

3. In areas of activation common to both WM and inhibitory demands of the task, practice effects occurred for phasic activity

Table 4

Regions of event-related activation for errors of commission

\begin{tabular}{|c|c|c|c|c|c|c|c|}
\hline \multirow[t]{2}{*}{ Structure } & \multirow[t]{2}{*}{ BA } & \multirow[t]{2}{*}{ HS } & \multirow[t]{2}{*}{ Vol $(\mu 1)$} & \multicolumn{3}{|c|}{ Center of mass } & \multirow[t]{2}{*}{ Performance effect? } \\
\hline & & & & $x$ & $y$ & $z$ & \\
\hline \multicolumn{8}{|l|}{ Frontal lobe } \\
\hline Middle frontal & 46 & $\mathrm{R}$ & 314 & -45.6 & 27.2 & 25.7 & * \\
\hline Inferior frontal & 9 & $\mathrm{~L}$ & 160 & -56.4 & 4.4 & 25.2 & $*$ \\
\hline \multirow[t]{2}{*}{ Anterior cingulate } & $24 / 32$ & BL & 791 & -0.6 & 28.8 & 34.1 & \\
\hline & 24 & $\mathrm{R}$ & 171 & -1.8 & 26.1 & 22.7 & \\
\hline Cingulate gyrus & $24 / 32$ & $\mathrm{R}$ & 143 & -10.4 & 9.9 & 33.0 & \\
\hline \multicolumn{8}{|l|}{ Subcortical } \\
\hline \multirow[t]{2}{*}{ Insula } & $13 / 47$ & $\mathrm{R}$ & 322 & -29.0 & 19.5 & -6.5 & \\
\hline & & $\mathrm{L}$ & 370 & 31.4 & 18.2 & -4.7 & \\
\hline
\end{tabular}

Positive values for $x, y$, and $z$ coordinates denote locations right, anterior, and superior relative to the anterior commissure. BA—Brodmann's area, HScerebral hemisphere. 
only, and these effects are congruent with the effects observed in those areas activated by the inhibitory demands of the task alone.

4. Practice and individual differences in task performance were associated with the ability to modulate and maintain activations in frontostriatal areas mediating attentional control.

5. Our findings suggest that the areas that differ between individuals can be modulated by practice within an individual and demonstrate that dissociable patterns of performancerelated brain activation, increased phasic, event-related activation, and decreased tonic activation, can be induced as a result of practice, in the same task, and in the same brain.

In the absence of practice-related changes in behavioral performance, there were substantial changes in the functional activations underlying task performance

In line with predictions and with previous neuroimaging studies of brief practice on WM tasks (Garavan et al., 2000; Jansma et al., 2001; Landau et al., 2004) tonic WM-related activations decreased over the course of both a single task block and over the duration of several task blocks. These decreases were seen in regions of the cingulate and posterior parietal cortex (PPC) over the course of a single block of trials but in dorso- and ventrolateral PFC, insular, striatal, thalamic, and brainstem areas from early to late in task performance. In contrast, quite a different pattern of results was observed for phasic inhibitory processes. Decreases were observed in the inhibitory network over the course of a single block of trials, but significant increases in dlPFC and inferior parietal cortex were observed during later task performance, relative to early task performance. Of the regions of activation common to both the WM and inhibitory requirements of the task, bilateral IFG/ insula and putamen showed practice-related changes in activation in phasic inhibitory activity only. Inhibitory activation in these areas decreased as a result of practice, with the exception of activation in the right IFG/insular cortex, which increased late, relative to early in task performance.

These practice-related changes in activation were observed in the absence of any behavioral effects of practice, a result that is perhaps unsurprising, considering the brief $(30 \mathrm{~min})$ experience participants had with the task and its high difficulty level (5 memory items). Importantly, the lack of behavioral effects of practice does not preclude a practice-based interpretation of the activation changes. Practice-related changes in activations without concomitant behavioral changes have been observed previously (e.g., Landau et al., 2004; Shadmehr and Holcomb, 1997). In addition, it has been emphasized that behavioral changes, such as decreased reaction times, can confound the interpretation of activation changes, which may be secondary to, rather than a source of, the behavioral changes (Poldrack, 2000; Sanes and Donoghue, 2000). The present study demonstrates that adaptive modulation of functional activations in common and unique task areas occurs as a result of task practice and enables the maintenance of successful inhibitory control for a sustained period of task performance. Functional activations were observed to change over time as a result of both itemspecific and task practice, but these changes were not dependent on nor reflected in the behzavioral changes usually associated with practice and learning.
In the context of different effects of item repetition and general task practice, there were distinct practice-related responses in areas underlying tonic working memory and phasic inhibitory processes

The present study employed a mixed block- and event-related design which enabled the separation of tonic WM-related activations from phasic inhibitory activations and allowed us to separately assess the effects of practice on each. In addition, the WMI paradigm enabled the separation of item-specific repetition effects from the effects of practice on general task processes. For WM activations, the effect of both item repetition and task practice was decreased activity. These decreases occurred in different areas of the brain, indicating that item repetition and general task practice impacted on dissociable WM processes. In contrast, while phasic inhibitory activations decreased in association with item repetition, inhibitory activations increased as a result of task practice. These effects occurred in broadly similar areas of the inhibitory network, indicating differential effects of practice on the same inhibitory processes. Notably, increased activation was observed in several areas which have been repeatedly implicated in inhibitory control processes in several neuroimaging studies (Aron et al., 2003a, 2004; Garavan et al., 1999), including dlPFC and parietal cortex. This suggests that modulation of activations in these areas enabled the maintenance of inhibitory control throughout a sustained period of task performance.

\section{Item-specific versus process-specific effects in working memory}

Both item repetition and general task practice were associated with decreased WM activations. However, these decreases occurred in different areas of the brain. Over the course of a single task block, item repetition was associated with decreased activations in left posterior parietal cortex (PPC), ACC and the corpus callosum and decreased deactivation in right somatosensory cortex and a posterior midline region. In contrast, examining the effects of task practice by comparing activation during the early period of task performance to that during the late period revealed decreased activation across a large, bilateral prefrontal, and subcortical network comprising areas of lateral PFC, insula, striatum, and thalamus as well as in the pre-SMA and brainstem areas. The difference in the locus of practice effects suggests that practice-related changes occur in distinct working memory processes as a result of item repetition, when compared to general task practice. This finding contrasts with those of Poldrack et al. (1998) and Poldrack and Gabrieli (2001), who investigated item repetition and skill-learning effects in a mirror reading task. They observed that repetition effects occurred in the same areas of the brain as skill learning, even when there was a reorganization of the functional anatomy of task performance as a result of skill acquisition. However, Poldrack et al. also observed substantial behavioral effects of both repetition and task practice, and they associated practice-related changes in the functional anatomy of the task to a switch in the cognitive processes involved in mirror reading, induced by increasing behavioral automaticity. Thus, it is unsurprising that in a complex cognitive task, in which there were no behavioral changes and for which there was no evidence of a change in the underlying cognitive processes, we observed a different effect. Where we did see an overlap in the locus of item repetition and task practice effects, it was for item repetition effects on inhibitory activations and task practice effects of tonic WM activation, suggesting a differential impact of item repetition on 
cognitive processes involved in the two demands of the WMI task. This suggestion is discussed further below.

Decreased WM activations as a result of item repetition is consistent with Jansma et al. (2001), who reported a general pattern of reduced activity in WM regions as a result of extensive (840 trials) practice on a verbal Sternberg task with a constant target/ nontarget memory set. Although less robust than the changes resulting from general task practice, we observed decreased activation in several areas as a result of item repetition. Activation decreased in left posterior parietal cortex (PPC), an area thought to underlie the temporary storage of phonological information (Baddeley, 2003; Jonides et al., 1998a). Repeated exposure to target (NOGO) and nontarget (GO) stimuli over the course of a single block of trials may have enabled more automatic retrieval of the working memory set due to repeated access, therefore reducing the demand on WM storage in PPC. Similarly, repeated experience with the same set of target/nontarget stimuli over a single block of trials may have diminished the need to distinguish between target and nontarget stimuli and their associated behavioral responses. This may have contributed to reduced activation in the ACC, consistent with theories positing a role for this area in conflict monitoring (Bunge et al., 2001; Braver et al., 2001; Carter et al., 1998; Botvinick et al., 2001). That is, as the same set of GO and NOGO stimuli were repeatedly encountered over the course of a single block of trials, increased knowledge of the identity of the GO and NOGO stimuli may have reduced the amount of conflict experienced on each trial, associated with decreased activation in the ACC. There were also decreases in a region of the posterior cingulate/corpus callosum, however, as little is known about BOLD sensitivity to white matter signals, we may not confidently interpret this finding.

The midline/posterior locus of activation decreases occurring as a result of item repetition is in contrast to the frontostriatal decreases occurring as a result of practice effects on general WM processes. Decreased activation in the frontostriatal network is consistent with the hypothesis that practice, even over a brief period, is associated with increased neural efficiency in the working memory network (Kelly and Garavan, 2005; Garavan et al., 2000). It is also consistent with current theories of cognitive control in WM, which hypothesize that the PFC and the striatum interact in the achievement of cognitive control (Casey et al., 2002; Miller and Cohen, 2001; Braver and Cohen, 2000; Braver et al., 2002; Frank et al., 2001). According to these models, the PFC actively maintains representations of task goals and other taskrelevant information and mediates control by biasing or modulating information processing in posterior brain regions in accordance with the represented goals. The striatum is hypothesized to act as an adaptive gating mechanism, the function of which is to regulate the influence of incoming stimuli on WM. The striatum achieves this by updating new information into WM when the gate is open and preventing irrelevant stimuli from interfering with existing information or task goals, thereby supporting their maintenance, when the gate is closed. Further specifying the role of PFC in WM, particular areas of lateral PFC have been associated with working memory maintenance (dlPFC — see Curtis and D'Esposito, 2003), rehearsal (ventrolateral, premotor-e.g., Awh et al., 1995; Paulesu et al., 1993; Smith et al., 1998) and interference control (dorso/ ventrolateral-e.g., Bunge et al., 2001; D'Esposito et al., 1999; Jonides et al., 1998b). Similarly, activation in the pre-SMA during WM tasks has been associated with the integration of representations, particularly under conditions of high WM demand (Linden et al., 2003), and with attention switching between WM contents or responses (Derrfuss et al., 2004; Li et al., 2004; Rushworth et al., 2002). We propose that, with practice, the WM processing operations associated with these cortical and subcortical activations became less demanding in terms of attentional resources and more efficient in their neural implementation, reflected in a relative decrease in the magnitude of activation (Kelly and Garavan, 2005; Poldrack, 2000). When task experience increased, less activation across the WM network was required in order to maintain the same level of performance.

The lack of a behavioral practice effect raises the possibility that there is an alternative explanation for the decreases in tonic activation observed, that of increasing participant fatigue due to time on task. However, when we examined tonic activation changes in a group of five participants whose accuracy disimproved from early to late in performance (see Results section these participants did not show activation decreases in those areas which demonstrated a practice effect. This finding suggests that fatigue cannot account for the decreases in tonic activation observed, and that these decreases are instead consistent with increasing neural efficiency in the WM network subserving task performance.

In addition to the predominant pattern of decreasing activation, decreasing deactivation was observed in right primary somatosensory cortex and a posterior midline/callosal region. These are among a number of areas which consistently demonstrate decreased activation during the performance of cognitive tasks, hypothesized to reflect attentional modulation of task-irrelevant processing during task performance (Shulman et al., 1997a,b; Staines et al., 2002). Decreasing deactivation in these "default system" (Raichle et al., 2001) areas has been observed as a consequence of practice and is likely to reflect a decreased requirement to suppress processing in task-irrelevant areas as task performance becomes more skilled (Raichle et al., 1994).

\section{Item-specific versus process-specific effects in inhibitory control}

Hester et al. (2004b) observed that successful inhibitory control despite increasing WM demands was achieved by increasing activation in the areas uniquely activated by the inhibitory demands of the task, rather than increasing activation solely in regions common to both $\mathrm{WM}$ and inhibition. The present study demonstrates a corollary of this finding: over the course of a single block of trials, repeated experience with the same set of target/ nontarget stimuli was associated with a reduced demand on both tonic WM and phasic inhibitory network activity. This practice effect is therefore a mirror image of the effect of increasing WM load in the Hester et al. study in which increasing WM load was associated with increased activations in uniquely inhibition-related activations. In contrast, general task practice, also associated with decreased tonic WM-related activations, was associated with decreased activation in premotor regions, but with increased activation in bilateral dIPFC and inferior parietal cortex from early to late in performance.

Successful inhibitions activated bilateral regions of inferior, ventrolateral PFC, insula, and striatum. Ventrolateral PFC, including Brodmann's areas 44, 45, and 47, is consistently identified as central to inhibitory control across a broad range of higher order cognitive tasks (Aron et al., 2004; Chambers et al., in press). Similarly, there is an increasing literature supporting a central role for the striatum in inhibitory control. In addition to the models of cognitive control in WM discussed above (e.g., Braver 
and Cohen, 2000; Casey et al., 2002; Miller and Cohen, 2001), clinical studies have demonstrated inhibitory deficits in a range of conditions involving the loss of corticostriatal pathways through degeneration of the striatum (e.g., Aron et al., 2003b; Casey et al., 1997; Cools et al., 2003; Lawrence et al., 1998; Rosenberg et al., 1996; Rubia et al., 2001a). Studies of the effects of deep-brain stimulation, employed as a treatment for Parkinson's disease, have observed that stimulation of the subthalamic nucleus (STN) impairs performance on tasks requiring inhibition or attentional control, including GO/NOGO, Stroop, and WM tasks, possibly through disruption of output pathways through the striatum to PFC (Hershey et al., 2004; Jahanshahi et al., 2000).

Striatal and insular activations have previously been associated with less prepared and consequently more effortful inhibitory control (Kelly et al., 2004), and additional, compensatory recruitment of the IFG, particularly in the left hemisphere, has also been observed in older adults during the performance of inhibitory tasks, including the GO/NOGO task (Langenecker et al., 2004; Nielson et al., 2002). We observed robust decreases in activation in the left IFG and insula, in association with item repetition over the duration of a single block of trials. The decreasing activity extended from the IFG though the insula and into the putamen, and there were additional activation decreases in the left cerebellum and inferior parietal cortex. We suggest that over the course of a block of trials, increased knowledge of the identity of the GO and NOGO stimuli gained through repetition of a constant target/nontarget set enabled less effortful accomplishment of inhibitory control, reflected in decreased activation across the inhibitory network. That is, over the course of a block of trials, the effect of item repetition was to make successful inhibitory control become less neurally demanding. Notably, these decreases were observed in the left hemisphere. Inhibitory control is strongly right-lateralized (e.g., Garavan et al., 1999; Rubia et al., 2003), and this finding adds further support to the suggestion that additional left hemisphere subcortical and cortical areas were recruited early in performance when inhibitions are highly demanding, but these supporting activations decreased with practice, in association with less effortful accomplishment of inhibitory control.

Item repetition was associated with increased activation posteriorly in the brain - in primary motor cortex, inferior parietal, inferior temporal, and occipital areas, over the course of a block of trials. Similar activation increases were observed by Poldrack et al. (1998) and Poldrack and Gabrieli (2001) who also examined skill acquisition and item repetition effects in learning to read mirrorreversed text. They associated increases in posterior parietal, occipital, and temporal areas with stored representation of the learned mirror-reversed stimuli. Increased activation in representational areas of posterior cortex (occipital cortex, inferior temporal cortex) has also been observed in perceptual or perceptuomotor learning tasks involving repeated experience with visual stimuli (e.g., Sakai et al., 1998; Schwartz et al., 2002; Sigman et al., 2005).

The effects of item repetition on phasic inhibitory activations stand in contrast to the effects of general task practice. Successful inhibitory control in the later task blocks was associated with increased activation in bilateral prefrontal and parietal cortices as a result of general task practice. In the Introduction, we outlined two possible effects of task practice. As repeated task experience reduced WM demands and increased neural efficiency in the WM network and thus, as more attentional resources became available to devote to inhibitory control, one might expect (1) decreased activation in regions activated by the inhibitory demands of the task, and common regions activated by both the WM and inhibitory demands of the task, as these areas demonstrated increased activation in response to increased WM demands in the Hester et al. (2004b) study. Alternatively, one might predict (2) increased activation in those areas activated during successful inhibitions as the additional attentional resources are devoted to inhibitory processes, so that successful inhibitory control can be maintained over a sustained period of task performance under conditions of high WM demands.

The results support hypothesis (2). While a reduction in WMrelated activations as a result of task practice suggests increased neural efficiency underlying WM processes, and a concomitant reduction in demands on attentional control, we observed a practice-related increase in phasic activations for successful inhibitions. Increased activation was observed in right dorsolateral prefrontal and premotor cortex, and bilateral parietal cortex, cortical areas which are repeatedly implicated in cognitive control processes in the neuroimaging literature. For example, the areas of increase in BA 9 are equivalent to the area identified as the inferior frontal junction (IFJ). According to Brass et al. (2005); Derrfuss et al. (2004, 2005), this area plays a pivotal role in cognitive control across a broad rage of tasks, specifically in the active maintenance of task representations (such as stimulus-response rules). Increased activation was evident in right inferior BA 44 and 45 , also hypothesized to be critical to attentional and inhibitory control processes (Aron et al., 2004). In addition, ventrolateral and dorsolateral $\mathrm{PFC}$, right premotor and inferior parietal cortex represent areas which have been consistently implicated across a number of inhibitory paradigms (Rubia et al., 2001b; Garavan et al., 1999; Bunge et al., 2001). Taken together, these findings support the hypothesis that practice-related increases in phasic inhibitory activations reflect increased activation of a network of core cognitive control regions, enabling the maintenance of attention and task goals, and the achievement of inhibitory control under conditions of high WM demand, and for a sustained period of task performance.

As was the case with the practice-related changes in tonic WMrelated activations, an alternative explanation for the increases in inhibitory activation may be proposed. Across the eight blocks of the experiment, the same stimuli were used as both targets and distractors, albeit not on immediately successive blocks of trials. This may have generated increasing levels of interference with time on task. However, proactive interference is typically associated with increased RT and decrease accuracy (e.g., Jonides et al., 1998b; D’Esposito et al., 1999; Mecklinger et al., 2003), performance changes which were not observed in the present study. Furthermore, no differences were found when we compared the increases in activation for the group of five participants who showed the greatest slowing from early to late in performance and a group of five participants who showed the greatest decrease in RT from early to late in performance, which suggests that increasing interference cannot explain those activation increases.

Furthermore, the practice-related increase in activation in the frontoparietal inhibitory network is consistent with the results of Olesen et al. (2004). They examined the effects of extensive practice on an $n$-back working memory task and observed practicerelated increases in activation in a frontoparietal network. As discussed in Kelly and Garavan (2005), there is some suggestion that the pattern of practice-related activation change observed by Olesen et al. could be described by an inverted-U-shaped function, with activation decreasing later following extensive practice, 
consistent with the results of another study of long-term practice on the $n$-back task (Hempel et al., 2004). Thus, we suggest that the practice-related increases in inhibitory control-related activations would also follow an inverted-U function with extensive practice and behavioral improvement. This suggestion awaits further study.

An exception to the general pattern of increased inhibitory activation was a robust decrease in activation in the right putamen. As mentioned earlier, increased striatal activations have previously been associated with less prepared and consequently more effortful inhibitory control (Kelly et al., 2004), and practicerelated decreases in cortical and subcortical areas suggest that while these areas are recruited early in performance, the requirement for their supporting activations decreases with practice. In the present study, it appears that there was a redistribution of activation within the inhibitory network, from the striatum, and less robustly, premotor cortex, to a frontoparietal network supporting inhibitory control.

An examination of the areas of activation common to both WM and inhibitory control demands of the WMI task reveals that there were no effects of practice on common tonic activations. The effects of practice were observed for common activation in phasic activity, and these are congruent with the effects observed in the task-unique inhibitory areas

Examining the loci of decreases in phasic inhibitory activations occurring as a result of item repetition and decreases in tonic WMrelated activity occurring as a result of task practice suggests that these decreases occurred in broadly similar areas of the brain. Indeed, when areas of overlap between phasic inhibitory and tonic working-memory related activation were examined, activation common to both demands was observed in left dlPFC and parietal cortex, the IFG/insula bilaterally, and in the right putamen, consistent with Hester et al. (2004b). In fact, when we compare the regions of overlap between WM and inhibitory activation in the present study with the common regions of activation observed by Hester et al., regions of overlap in right inferior prefrontal and left parietal cortex are identical to both studies, providing further evidence that inferior PFC (ventrolateral/insula, BA 47/13) and parietal cortex are centrally involved in interference resolution or inhibitory processes common to both WM and inhibitory control.

Examining practice effects in the common areas of activation revealed an effect for phasic inhibitory processes only. The practice-related changes in common activations were congruent with those observed for unique inhibitory activations. Phasic activation in left IFG/insula, inferior parietal cortex and the right putamen decreased over the course of a block of trials, and also from early to late in task performance in the putamen. We have suggested that a practice-related increase in the neural efficiency of WM processes was reflected in a reduction in WM-related activations. The decrease in activations common to both WM and inhibitory processes indicates that practice was also associated with a reduction in demands on common attentional resources. This finding supports the hypothesis that a reduction in the WM demand on common neural resources allowed for the devotion of additional attentional resources to inhibitory processes, thereby increasing activation in the inhibitory network, and enabling the maintenance of successful inhibitory control over a sustained period of task performance under conditions of high WM demands.

Standing apart from this pattern of phasic decreases was an activation increase in the region of right IFG/insula common to both WM and inhibitory processes from early to late in task performance. Finding common activation in this area is unsurprising, and we have drawn attention to the centrality of this region to inhibitory control across a broad range of tasks, as discussed by Aron et al. (2004). Increases in phasic activation in this area suggests that demands on the inhibitory functions it subserves were increased as a result of task practice. The analysis of individual differences in inhibitory performance sheds further light on this finding, and suggests that differences in the ability to modulate inhibitory activations in this area, along with other task-specific inhibitory areas, predicted performance on the WMI task.

Individual differences in task performance were associated with the ability to modulate and maintain activations in frontostriatal areas mediating attentional control

In a recent paper, Liston et al. (2005) linked frontostriatal connectivity to developmental and individual differences in the efficient recruitment of cognitive control. When we examined individual differences in activation for successful inhibitions based on overall inhibitory performance, we observed that better performance was associated with higher levels of activation in right dlPFC and left inferior frontal/insular cortex, extending to the putamen. There were interactions between practice and performance in two areas of right inferior frontal cortex and insula, which indicated that while more accurate performers maintained a high level of activation in these areas, less accurate performers showed decreases in activation. These individual differences were observed for phasic inhibitory activations only-no such effects were seen for either tonic WM-related activations or regions of common activation. For errors of commission, two areas, in left and right lateral PFC, also showed increased error-related activation for better, relative to poorer performers.

In contrast to increases in frontostriatal areas demonstrated by better performers, poorer performance was associated with greater activation in right inferior parietal cortex. A possible source of this performance-based dissociation is the use of different cognitive strategies in performance of the WMI task. Glabus et al. (2003), in a study of individual differences in $n$-back working memory performance, also observed a dissociation in WM activations, with increased right frontoparietal activity in a high performing group but increased left hemisphere frontoparietal activation in a low performing group, a dissociation they attributed to differences in cognitive strategy.

The attentional control required on NOGO trials in the WMI task may be likened to the interference control required in the $n$ back task and may involve similar processes. An even greater similarity between the two tasks emerges in a variation of the $n$ back paradigm which includes lure trials - trials which match a recently seen stimulus, but not the critical $n$-back stimulus. When Gray et al. (2003) examined individual differences in the ability to inhibit interfering lure stimuli in an $n$-back working memory task, they observed that participants with high scores on a test of generalized fluid intelligence $(\mathrm{gF})$ were more accurate at rejecting lures and also showed greater activation in lateral PFC and parietal cortex on the lure trials. They interpreted this finding as reflecting high gF individuals' superior ability to control attention in order to protect goals or other information held in WM from interference and, at a neurocognitive level, increased frontoparietal activation reflecting this greater attentional control. Thus, we suggest that more successful performers may have relied on frontostriatal control processes, while poorer performers relied less on these frontostriatal attentional control processes and more on stimulus- 
driven representation retrieval, or on alternate, and less successful, attentional control processes subserved by parietal cortex.

Individual differences were observed in areas which demonstrated practice-related changes in phasic inhibitory activation and suggest that the areas that differ between individuals can be modulated by practice within an individual

We have put forward a hypothesis that a reduced WM demand on common neural resources, occurring as a result of general task practice, allowed for the devotion of additional attentional resources to inhibitory processes. This was reflected in increasing activation in the inhibitory network, so that successful inhibitory control could be maintained over a sustained period of task performance under conditions of high WM demands. Among the areas which demonstrated this increase in inhibitory activations were the areas which showed greater activation in better performers, relative to poorer performers: right dorsolateral prefrontal and inferior frontal cortex, and also an area of right IFG/insula common to both WM and inhibitory processes. These prefrontal areas showed increased activation in association with increasing WM load in the study by Hester et al. (2004b). Taken together, these findings support our earlier interpretations of separable effects of item and task repetition on inhibitory control processes and suggest that both task practice and superior performance on the WMI task are associated with the modulation of activation in dorsolateral prefrontal and inferior frontal/insular cortex. This is consistent with Hester et al. (2004b) who observed that individual differences in activity in the right dlPFC predicted inhibitory performance, as well as a number of other studies demonstrating individual differences in the ability to modulate neural activations under conditions of high cognitive demands (e.g., Kane and Engle, 2002; Mecklinger et al., 2003; Bunge et al., 2001; Gray et al., 2003). Interestingly, similar areas in bilateral dlPFC also showed increased error-related activation for better, relative to poorer performers, consistent with a recent meta-analysis of error-related activations in GO/NOGO tasks (Hester et al., 2004a). In that metaanalysis, participants thought to show greater attention to the task, as indicated by slower RTs and low scores on a measure of absentmindedness (Broadbent et al., 1982) also showed higher levels of error-related activation in lateral $\mathrm{PFC}$, relative to participants with faster RTs and higher absentmindedness scores.

A comparable effect was observed in the large bilateral IFG/ insular and striatal clusters. The left cluster showed an overall performance effect-with greater activation for better performers, while the right cluster showed an interaction, such that better performers maintained a consistent level of activation in this area over the course of a block of trials, while poorer performers showed decreases in activation. Both of these regions showed an overall practice-related decrease in activation over the course of a block of trials, which we have suggested reflects the recruitment of these areas under conditions of more effortful inhibitory control (e.g., Kelly et al., 2004; Langenecker et al., 2004), as occurs early in practice, but less effortful and less neurally demanding accomplishment of inhibitory control as a result of item repetition. Thus, performance effects in these areas reveal that maintaining activity benefited performance overall but not over the course of a block, so that activity decreased generally across the sample, but was slightly higher in the better performers.

Taken together, these findings suggest that areas which differ in activation between individuals on the basis of their performance on a complex cognitive task can be modulated by practice within an individual. These areas, comprising dlPFC, IFG/insula and the striatum, are central to flexible cognitive control (Casey et al., 2002; Miller and Cohen, 2001; Braver and Cohen, 2000; Braver et al., 2002; Frank et al., 2001). In particular, neural activity in dIPFC is hypothesized to underlie individual differences in the ability to exert executive control over attention, general fluid intelligence, and consequently, individual differences in performance across a wide range of tasks that place a demand on cognitive control (Duncan, 1995, 2003; Duncan et al., 2000; Kane, 2003; Kane and Engle, 2002; Engle and Kane, 2004). Single cell and human neuroimaging studies demonstrate how this is possible. Studies have shown that the response properties of dlPFC cells are highly adaptable - cells in dIPFC respond to different kinds of input and to code different types of information across a wide range of tasks (Miller and Cohen, 2001; Miller et al., 2002; Duncan, 2001). Our findings suggest that areas thought to underlie individual differences in flexible cognitive capabilities, associated with successful interaction within our complex world and adaptation to novel situations, are subject to modulation by practice. This raises the possibility that this fundamental cognitive flexibility is amenable to practice and because it is an ability purported to underlie performance across a wide range of cognitively demanding tasks, practice-related improvements in one task might therefore translate to improvements on other, similarly demanding tasks, and even to an improvement of $\mathrm{gF}$. Single-cell studies in nonhuman primates have already suggested how practice may affect these adaptive cells - as an animal becomes more practiced on the task, the number of cells responding decreases, and those that respond have more narrowly tuned response properties (Rainer and Miller, 2000; Asaad et al., 1998).

The present study has demonstrated that activity in areas of the brain thought to explain individual differences in general intelligence and performance across a wide range of tasks is subject to modulation by practice. More specifically, general task practice has the effect of modulating activations so that they become more similar to activations demonstrated by superior performers. This finding is consistent with several recent studies investigating the interactions between individual differences, dopamine (DA) activity in PFC, and training. For example, the observation that practice makes activations more similar to those of better performers is analogous to the results of a study which examined the effects of attentional training on performance and brain activations (measured by EEG) in children (Rueda et al., 2005). Rueda et al. demonstrated that training improved attention in younger children in a manner similar to increasing age. Furthermore, attention training produced an EEG pattern in younger children similar to that of the older children, and in older children, an EEG pattern similar to that shown by adults. The effects of the present study are also paralleled in a recent examination of the effects of a dopamine (DA) D2 receptor stimulant (bromocriptine) on WM performance and PFC activation in participants identified as having either a high or low WM span (Gibbs and D'Esposito, 2005). In low span participants, administration of the DA agonist increased performance and decreased activation in PFC during the probe period of a WM task, so that it was more like basal activation in the high span participants. That is, DA stimulation in low span participants made both their brain activation and performance more like that of high span participants. Individual differences in WM span and underlying PFC function are, at least in part, related to genetic differences, particularly, the possession of certain polymorphisms of genes which impact on DA 
activity in PFC (such as COMT, MAOA, DRD4, and DAT-1, e.g., Blasi et al., 2005; Diamond et al., 2004; Fan et al., 2003; Fossella et al., 2002; Greenwood and Parasuraman, 2003; Parasuraman et al., 2005). For example, in the Rueda et al. (2005) study, children with the pure long homozygous DAT-1 allele showed better performance on tests of attention and intelligence than those with the heterozygous alleles.

An important point to note is that the practice- and individualdifference-related modulation of phasic inhibitory activations in the present study corresponded to increasing activation, a result that may appear at odds with the neural efficiency framework, which suggests that better performers, higher intelligence $(\mathrm{gF})$ and practice are associated with decreased neural activations, reflective of greater neural efficiency in the brain networks underlying performance (e.g., Deary, 2000; Haier et al., 1992a,b; Neubauer et al., 2004). However, the association between decreased neural activations and superior performance is typically seen for tonic, sustained activations, and higher levels of phasic, event-related activation have been observed to be embedded in lower overall levels of activation in the brains of better performers (Rypma et al., 2002). The findings of the present study are consistent with these observations. In this study, increased phasic inhibitory activations were associated with both practice and better performance on the WMI task, consistent with those previous studies which have related increased phasic, eventrelated activations to superior performance, particularly under demanding conditions (e.g., Bunge et al., 2001; Gray et al., 2003; Rypma and D'Esposito, 1999). Decreased WM activations, reflective of increased neural efficiency in the WM network, were also observed as a result of practice. While no performance-related differences in tonic activations were observed, this pattern is consistent with those studies relating increased neural efficiency (lower tonic activation) to both better performance and practice (e.g., Beauchamp et al., 2003; Buchel et al., 1999; Garavan et al., 2000; Haier et al., 1992a,b), and those studies which demonstrate that DA administration improves performance and decreases tonic activations in PFC in individuals with low basal WM capacity (low DA activity_Egan et al., 2001; Mattay et al., 2002, 2003; Mehta et al., 2000). Thus, the present study has demonstrated how practice can induce both patterns of performance-related brain activationincreased phasic, event-related activation and decreased tonic activation, in the same task, and in the same brain. As our findings illustrate, the relationships that exist between individual differences, performance, activation, neural efficiency, and practice are intriguing and merit considerable further investigation.

\section{Conclusions}

The present study represents a novel examination of practice effects on a complex cognitive task in terms of the separation of item-specific and general task processes. In the context of different effects of item repetition and general task practice, there were distinct practice-related responses in areas underlying tonic working memory and phasic inhibitory processes. These data demonstrate that with both item- and process-specific practice, WM processing operations become less demanding in terms of attentional resources and more efficient in their neural implementation, reflected in a relative decrease in the magnitude of activations. In contrast, while phasic inhibitory activations decreased in association with item repetition, inhibitory activations increased as a result of task practice. These changes reflected decreases in supportive, left hemisphere cortical and subcortical activations as a result of item repetition, and modulation of activations in predominantly right hemisphere frontoparietal cortex, which enabled the maintenance of inhibitory control throughout a sustained period of task performance. An examination of the areas of activation common to both WM and inhibitory control demands of the WMI task suggested that the practicerelated decrease in the neural demands of WM processes enabled the devotion of attentional resources to the inhibitory demands of the task, reflected in increased activations in frontostriatal and parietal central to inhibitory control processes. Finally, both practice and individual differences in task performance were associated with the ability to modulate and maintain activations in frontostriatal areas mediating attentional control, suggesting that the areas that differ between individuals can be modulated by practice within an individual. Thus, the present study has demonstrated how practice can induce both patterns of performance-related brain activation-increased phasic, event-related activation, and decreased tonic activation, in the same task, and in the same brain, similar to the effect previously observed by Rypma et al. (2002). These findings are consistent with the literature on individual differences in performance and their relation to functional activations and emphasize the potentially important influence of performance differences on activations and practice effects. These results suggest the possibility that the ability to control attention, thought to underlie individual differences in general fluid intelligence, is amenable to practice.

The present study provides support for theories emphasizing the central role of the striatum in flexible cognitive control processes (e.g., Braver and Cohen, 2000; Casey et al., 2002). Increasingly, the striatum has been identified as a region important in the ability to alter behavior in novel or changing conditions, due to its role in providing information for subsequent cortical processing (Jahanshahi et al., 2000; Troyer et al., 2004). In particular, these effects have been associated with response-related processes (Aron et al., 2003b; Manoach et al., 2003), providing a basis on which further examination of the contribution of the striatum/basal ganglia to executive control functions may be conducted. The implication of frontostriatal circuitry in progressive illnesses marked by severe cognitive deficits, including Parkinson's and Huntington's diseases, as well as a range of other clinical conditions such as ADHD, schizophrenia, and OCD, highlights the need for further research into the role of the striatum in cognitive control and learning processes.

\section{Acknowledgments}

This research was supported by USPHS grant DA14100 to Garavan and NIMH grants MH63434 and MH65350 to Foxe. The assistance of Raj Sangoi is gratefully acknowledged. A grant from the Centre for Advanced Brain Imaging (CABI) at the Nathan Kline Institute provided magnet time for this study.

\section{References}

Aron, A.R., Fletcher, P.C., Bullmore, E.T., Sahakian, B.J., Robbins, T.W., 2003a. Stop-signal inhibition disrupted by damage to right inferior frontal gyrus in humans. Nat. Neurosci. 6 (2), 115-116.

Aron, A.R., Watkins, L., Sahakian, B.J., Monsell, S., Barker, R.A., Robbins, T.W., 2003b. Task-set switching deficits in early-stage 
Huntington's disease: implications for basal ganglia function. J. Cogn. Neurosci. 15 (5), 629-642.

Aron, A.R., Robbins, T.W., Poldrack, R.A., 2004. Inhibition and the right inferior frontal cortex. Trends Cogn. Sci. 8 (4), 170-177.

Asaad, W.F., Rainer, G., Miller, E.K., 1998. Neural activity in the primate prefrontal cortex during associative learning. Neuron 21 (6), $1399-1407$.

Awh, E., Smith, E.E., Jonides, J., 1995. Human rehearsal processes and the frontal lobes: PET evidence. Ann. N. Y. Acad. Sci. 769, 97-117.

Baddeley, A., 2003. Working memory: looking back and looking forward. Nat. Rev., Neurosci. 4 (10), 829-839.

Baddeley, A., Hitch, G.J., 1974. Working memory. In: Bower, G.H. (Ed.), The Psychology of Learning and Motivation, vol. 8. Academic Press, London, pp. 47-90.

Baddeley, A., Chincotta, D., Adlam, A., 2001. Working memory and the control of action: evidence from task switching. J. Exp. Psychol. Gen. 130 (4), 641-657.

Beauchamp, M.H., Dagher, A., Aston, J.A., Doyon, J., 2003. Dynamic functional changes associated with cognitive skill learning of an adapted version of the Tower of London task. NeuroImage 20 (3), 1649-1660.

Blasi, G., Mattay, V.S., Bertolino, A., Elvevag, B., Callicott, J.H., Das, S., Kolachana, B.S., Egan, M.F., Goldberg, T.E., Weinberger, D.R., 2005. Effect of catechol- $O$-methyltransferase val158met genotype on attentional control. J. Neurosci. 25 (20), 5038-5045.

Botvinick, M.M., Braver, T.S., Barch, D.M., Carter, C.S., Cohen, J.D., 2001. Conflict monitoring and cognitive control. Psychol. Rev. 108 (3), $624-652$.

Brass, M., Derrfuss, J., Forstmann, B., von Cramon, D.Y., 2005. The role of the inferior frontal junction area in cognitive control. Trends Cogn. Sci. 9 (7), 314-316.

Braver, T.S., Cohen, J.D., 2000. On the control of control: dopamine in regulating prefrontal function and working memory. In: Monsell, S., Driver, J. (Eds.), Attention and Performance XVIIIMIT Press, Cambridge, MA, pp. 713-737.

Braver, T.S., Barch, D.M., Gray, J.R., Molfese, D.L., Snyder, A., 2001. Anterior cingulate cortex and response conflict: effects of frequency, inhibition and errors. Cereb. Cortex 11 (9), 825-836.

Braver, T.S., Barch, D.M., Cohen, J.D., 2002. The role of prefrontal cortex in normal and disordered cognitive control: a cognitive neuroscience perspective. In: Stuss, D.T., Knight, R.T. (Eds.), Principles of Frontal Lobe Function. Oxford Univ. Press, Oxford, pp. 428-447.

Braver, T.S., Reynolds, J.R., Donaldson, D.I., 2003. Neural mechanisms of transient and sustained cognitive control during task switching. Neuron 39 (4), 713-726.

Broadbent, D.E., Cooper, P.F., Fitzgerald, P., Parkes, K.R., 1982. The Cognitive Failures Questionnaire (CFQ) and its correlates. Br. J. Clin. Psychol. 21 (1), 1-16.

Buchel, C., Coull, J.T., Friston, K.J., 1999. The predictive value of changes in effective connectivity for human learning. Science 283 (5407), $1538-1541$.

Bunge, S.A., Ochsner, K.N., Desmond, J.E., Glover, G.H., Gabrieli, J.D., 2001. Prefrontal regions involved in keeping information in and out of mind. Brain 124 (Pt. 10), 2074-2086.

Bunting, M.F., Conway, A.R.A., Heitz, R.P., 2004. Individual differences in the fan effect and working memory capacity. J. Mem. Lang. 51 (4), 604-622.

Carter, C.S., Braver, T.S., Barch, D.M., Botvinick, M.M., Noll, D., Cohen, J.D., 1998. Anterior cingulate cortex, error detection, and the online monitoring of performance. Science 280 (5364), 747-749.

Casey, B.J., Castellanos, F.X., Giedd, J.N., Marsh, W.L., Hamburger, S.D., Schubert, A.B., Vauss, Y.C., Vaituzis, A.C., Dickstein, D.P., Sarfatti, S.E., Rapoport, J.L., 1997. Implication of right frontostriatal circuitry in response inhibition and attention-deficit/hyperactivity disorder. J. Am. Acad. Child Adolesc. Psych. 36 (3), 374-383.

Casey, B.J., Tottenham, N., Fossella, J., 2002. Clinical, imaging, lesion, and genetic approaches toward a model of cognitive control. Dev. Psychobiol. 40 (3), 237-254.
Chambers, C.D., Bellgrove, M.A., Stokes, M.G., Henderson, T.R., Garavan, H., Robertson, I.H., Mattingley, J., in press. Executive 'brake' failure following deactivation of human frontal lobe. J. Cogn. Neurosci.

Conway, A.R.A., Cowan, N., Bunting, M.F., 2001. The cocktail party phenomenon revisited: the importance of working memory capacity. Psychon. Bull. Rev. 8 (2), 331-335.

Conway, A.R.A., Kane, M.J., Engle, R.W., 2003. Working memory capacity and its relation to general intelligence. Trends Cogn. Sci. 7 (12), 547-552.

Cools, R., Barker, R.A., Sahakian, B.J., Robbins, T.W., 2003. L-Dopa medication remediates cognitive inflexibility, but increases impulsivity in patients with Parkinson's disease. Neuropsychologia 41 (11), $1431-1441$.

Cowan, N., 2001. The magical number 4 in short-term memory: a reconsideration of mental storage capacity. Behav. Brain Sci. 24 (1), $87+$.

Curtis, C.E., D'Esposito, M., 2003. Persistent activity in the prefrontal cortex during working memory. Trends Cogn. Sci. 7 (9), 415-423.

Deary, I.J., 2000. Looking Down on Human Intelligence: From Psychometrics to the Brain. Oxford Univ. Press.

de Fockert, J.W., Rees, G., Frith, C.D., Lavie, N., 2001. The role of working memory in visual selective attention. Science 291 (5509), 1803-1806.

Derrfuss, J., Brass, M., von Cramon, D.Y., 2004. Cognitive control in the posterior frontolateral cortex: evidence from common activations in task coordination, interference control, and working memory. NeuroImage 23 (2), 604-612.

Derrfuss, J., Brass, M., Neumann, J., von Cramon, D.Y., 2005. Involvement of the inferior frontal junction in cognitive control: meta-analyses of switching and Stroop studies. Hum. Brain Mapp. 25 (1), 22-34.

D’Esposito, M., Postle, B.R., Jonides, J., Smith, E.E., 1999. The neural substrate and temporal dynamics of interference effects in working memory as revealed by event-related functional MRI. Proc. Natl. Acad. Sci. U. S. A. 96 (13), 7514-7519.

Diamond, A., Briand, L., Fossella, J., Gehlbach, L., 2004. Genetic and neurochemical modulation of prefrontal cognitive functions in children. Am. J. Psychiatry 161 (1), 125-132.

Duncan, J., 1995. Attention, intelligence and the frontal lobes. In: Gazzaniga, M.S. (Ed.), The Cognitive Neurosciences. MIT Press, Cambridge, MA, pp. 721-733.

Duncan, J., 2001. An adaptive coding model of neural function in prefrontal cortex. Nat. Rev., Neurosci. 2 (11), 820-829.

Duncan, J., 2003. Intelligence tests predict brain response to demanding task events. Nat. Neurosci. 6 (3), 207-208.

Duncan, J., Seitz, R.J., Kolodny, J., Bor, D., Herzog, H., Ahmed, A., Newell, F.N., Emslie, H., 2000. A neural basis for general intelligence. Science 289 (5478), 457-460.

Egan, M.F., Goldberg, T.E., Kolachana, B.S., Callicott, J.H., Mazzanti, C.M., Straub, R.E., Goldman, D., Weinberger, D.R., 2001. Effect of COMT Val108/158 Met genotype on frontal lobe function and risk for schizophrenia. Proc. Natl. Acad. Sci. U. S. A. 98 (12), 6917-6922.

Engle, R.W., Kane, M.J., 2004. Executive attention, working memory capacity, and a two-factor theory of cognitive control. Psychol. Learn. Motiv. Adv. Res. Theory 44, 145-199.

Engle, R.W., Tuholski, S.W., Laughlin, J.E., Conway, A.R., 1999. Working memory, short-term memory, and general fluid intelligence: a latentvariable approach. J. Exp. Psychol. Gen. 128 (3), 309-331.

Fan, J., Fossella, J., Sommer, T., Wu, Y., Posner, M.I., 2003. Mapping the genetic variation of executive attention onto brain activity. Proc. Natl. Acad. Sci. U. S. A. 100 (12), 7406-7411.

Fossella, J., Sommer, T., Fan, J., Wu, Y., Swanson, J.M., Pfaff, D.W., Posner, M.I., 2002. Assessing the molecular genetics of attention networks. BMC Neurosci. 3, 14.

Frank, M.J., Loughry, B., O’Reilly, R.C., 2001. Interactions between frontal cortex and basal ganglia in working memory: a computational model. Cogn. Affect. Behav. Neurosci. 1 (2), 137-160.

Garavan, H., Ross, T.J., Stein, E.A., 1999. Right hemispheric dominance of 
inhibitory control: an event-related functional MRI study. Proc. Natl. Acad. Sci. U. S. A. 96 (14), 8301-8306.

Garavan, H., Kelley, D., Rosen, A., Rao, S.M., Stein, E.A., 2000. Practicerelated functional activation changes in a working memory task. Microsc. Res. Tech. 51 (1), 54-63.

Gevins, A., Smith, M.E., McEvoy, L., Yu, D., 1997. High-resolution EEG mapping of cortical activation related to working memory: effects of task difficulty, type of processing, and practice. Cereb. Cortex 7 (4), $374-385$.

Gibbs, S.E., D'Esposito, M., 2005. Individual capacity differences predict working memory performance and prefrontal activity following dopamine receptor stimulation. Cogn. Affect. Behav. Neurosci. 5 (2), $212-221$.

Glabus, M.F., Horwitz, B., Holt, J.L., Kohn, P.D., Gerton, B.K., Callicott, J.H., Meyer-Lindenberg, A., Berman, K.F., 2003. Interindividual differences in functional interactions among prefrontal, parietal and parahippocampal regions during working memory. Cereb. Cortex 13 (12), 1352-1361

Gray, J.R., Chabris, C.F., Braver, T.S., 2003. Neural mechanisms of general fluid intelligence. Nat. Neurosci. 6 (3), 316-322.

Greenwood, P.M., Parasuraman, R., 2003. Normal genetic variation, cognition, and aging. Behav. Cogn. Neurosci. Rev. 2 (4), 278-306.

Haier, R.J., Siegel, B.V., MacLachlan, A., Soderling, E., 1992 a. Regional glucose metabolic changes after learning a complex visuospatial/motor task: a positron emission tomographic study. Brain Res. 570 (1), 134-143.

Haier, R.J., Siegel, B.V., Tang, C., Abel, L., 1992b. Intelligence and changes in regional cerebral glucose metabolic rate following learning. Intelligence 16 (3), 415-426.

Hempel, A., Giesel, F.L., Caraballo, N.M.G., Amann, M., Meyer, H., Wustenberg, T., Essig, M., Schroeder, J., 2004. Plasticity of cortical activation related to working memory during training. Am. J. Psychiatry 161 (4), 745-747.

Hershey, T., Revilla, F.J., Wernle, A., Gibson, P.S., Dowling, J.L., Perlmutter, J.S., 2004. Stimulation of STN impairs aspects of cognitive control in PD. Neurology 62 (7), 1110-1114.

Hester, R., Garavan, H., 2005. Working memory and executive function: the influence of content and load on the control of attention. Mem. Cogn. 33 (2), 221-233.

Hester, R., Fassbender, C., Garavan, H., 2004a. Individual differences in error processing: a review and reanalysis of three event-related fMRI studies using the GO/NOGO task. Cereb. Cortex 14 (9), 986-994.

Hester, R., Murphy, K., Garavan, H., 2004b. Beyond common resources: the cortical basis for resolving task interference. NeuroImage 23 (1), $202-212$.

Holroyd, C.B., Nieuwenhuis, S., Yeung, N., Nystrom, L., Mars, R.B., Coles, M.G., Cohen, J.D., 2004. Dorsal anterior cingulate cortex shows fMRI response to internal and external error signals. Nat. Neurosci. 7 (5), 497-498.

Jahanshahi, M., Ardouin, C.M.A., Brown, R.G., Rothwell, J.C., Obeso, J., Albanese, A., Rodriguez-Oroz, M.C., Moro, E., Benabid, A.L., Pollak, P., Limousin-Dowsey, P., 2000. The impact of deep brain stimulation on executive function in Parkinson's disease. Brain 123 (6), $1142-1154$.

Jansma, J.M., Ramsey, N.F., Slagter, H.A., Kahn, R.S., 2001. Functional anatomical correlates of controlled and automatic processing. J. Cogn. Neurosci. 13 (6), 730-743.

Jonides, J., Schumacher, E.H., Smith, E.E., Koeppe, R.A., Awh, E., Reuter-Lorenz, P.A., Marshuetz, C., Willis, C.R., 1998a. The role of parietal cortex in verbal working memory. J. Neurosci. 18 (13), $5026-5034$.

Jonides, J., Smith, E.E., Marshuetz, C., Koeppe, R.A., Reuter-Lorenz, P.A., 1998b. Inhibition in verbal working memory revealed by brain activation. Proc. Natl. Acad. Sci. U. S. A. 95 (14), 8410-8413.

Kane, M.J., 2003. The intelligent brain in conflict. Trends Cogn. Sci. 7 (9), $375-377$

Kane, M.J., Engle, R.W., 2000. Working-memory capacity, proactive interference, and divided attention: Limits on long-term memory retrieval. J. Exper. Psychol., Learn., Mem., Cogn. 26 (2), 336-358

Kane, M.J., Engle, R.W., 2002. The role of prefrontal cortex in working-memory capacity, executive attention, and general fluid intelligence: an individual-differences perspective. Psychon. Bull. Rev. 9 (4), 637-671.

Kane, M.J., Engle, R.W., 2003. Working-memory capacity and the control of attention: the contributions of goal neglect, response competition, and task set to Stroop interference. J. Exp. Psychol. Gen. 132 (1), 47-70.

Kane, M.J., Bleckley, M.K., Conway, A.R., Engle, R.W., 2001. A controlled-attention view of working-memory capacity. J. Exp. Psychol. Gen. 130 (2), 169-183.

Kane, M.J., Hambrick, D.Z., Tuholski, S.W., Wilhelm, O., Payne, T.W., Engle, R.W., 2004. The generality of working memory capacity: a latent-variable approach to verbal and visuospatial memory span and reasoning. J. Exp. Psychol. Gen. 133 (2), 189-217.

Kelly, A.M.C., Garavan, H., 2005. Human functional neuroimaging of brain changes associated with practice. Cereb. Cortex 15 (8), 1089-1102.

Kelly, A.M.C., Hester, R., Murphy, K., Javitt, D.C., Foxe, J.J., Garavan, H., 2004. Prefrontal-subcortical dissociations underlying inhibitory control revealed by event-related fMRI. Eur. J. Neurosci. 19 (11), 3105-3112.

Keppel, G., 1991. Design and Analysis: A Researcher's Handbook, 3rd ed. Prentice Hall, Englewood Cliffs, NJ.

Kiehl, K.A., Liddle, P.F., Hopfinger, J.B., 2000. Error processing and the rostral anterior cingulate: an event-related fMRI study. Psychophysiology 37 (2), 216-223.

Landau, S.M., Schumacher, E.H., Garavan, H., Druzgal, T.J., D'Esposito, M., 2004. A functional MRI study of the influence of practice on component processes of working memory. NeuroImage 22 (1), 211-221.

Langenecker, S.A., Nielson, K.A., Rao, S.M., 2004. fMRI of healthy older adults during Stroop interference. NeuroImage 21 (1), 192-200.

Lawrence, A.D., Sahakian, B.J., Robbins, T.W., 1998. Cognitive functions and corticostriatal circuits: insights from Huntington's disease. Trends Cogn. Sci. 2 (10), 379-388.

Li, Z.H., Sun, X.W., Wang, Z.X., Zhang, X.C., Zhang da, R., He, S., Hu, X.P., 2004. Behavioral and functional MRI study of attention shift in human verbal working memory. NeuroImage 21 (1), 181-191.

Liddle, P.F., Kiehl, K.A., Smith, A.M., 2001. Event-related fMRI study of response inhibition. Hum. Brain Mapp. 12 (2), 100-109.

Linden, D.E.J., Bittner, R.A., Muckli, L., Waltz, J.A., Kriegeskorte, N., Goebel, R., Singer, W., Munk, M.H.J., 2003. Cortical capacity constraints for visual working memory: dissociation of fMRI load effects in a fronto-parietal network. NeuroImage 20 (3), 1518-1530.

Liston, C., Watts, R., Tottenham, N., Davidson, M.C., Niogi, S., Ulug, A.M., Casey, B.J., 2005. Frontostriatal microstructure modulates efficient recruitment of cognitive control. Cereb. Cortex. doi:10.1093/ cercor/bhj003.

Manoach, D.S., Schlaug, G., Siewert, B., Darby, D.G., Bly, B.M., Benfield, A., Edelman, R.R., Warach, S., 1997. Prefrontal cortex fMRI signal changes are correlated with working memory load. NeuroReport 8 (2), $545-549$.

Manoach, D.S., Greve, D.N., Lindgren, K.A., Dale, A.M., 2003. Identifying regional activity associated with temporally separated components of working memory using event-related functional MRI. NeuroImage 20 (3), 1670-1684.

Mattay, V.S., Tessitore, A., Callicott, J.H., Bertolino, A., Goldberg, T.E., Chase, T.N., Hyde, T.M., Weinberger, D.R., 2002. Dopaminergic modulation of cortical function in patients with Parkinson's disease. Ann. Neurol. 51 (2), 156-164.

Mattay, V.S., Goldberg, T.E., Fera, F., Hariri, A.R., Tessitore, A., Egan, M.F., Kolachana, B., Callicott, J.H., Weinberger, D.R., 2003. Catechol $O$-methyltransferase val158-met genotype and individual variation in the brain response to amphetamine. Proc. Natl. Acad. Sci. U. S. A. 100 (10), 6186-6191.

McEvoy, L.K., Smith, M.E., Gevins, A., 1998. Dynamic cortical networks 
of verbal and spatial working memory: effects of memory load and task practice. Cereb. Cortex 8 (7), 563-574.

Mecklinger, A., Weber, K., Gunter, T.C., Engle, R.W., 2003. Dissociable brain mechanisms for inhibitory control: effects of interference content and working memory capacity. Brain Res. Cogn. Brain Res. 18 (1), $26-38$.

Mehta, M.A., Owen, A.M., Sahakian, B.J., Mavaddat, N., Pickard, J.D., Robbins, T.W., 2000. Methylphenidate enhances working memory by modulating discrete frontal and parietal lobe regions in the human brain. J. Neurosci. 20 (6), RC65.

Miller, E.K., Cohen, J.D., 2001. An integrative theory of prefrontal cortex function. Annu. Rev. Neurosci. 24, 167-202.

Miller, E.K., Freedman, D.J., Wallis, J.D., 2002. The prefrontal cortex: categories, concepts and cognition. Philos. Trans. R. Soc. London, Ser. B Biol. Sci. 357 (1424), 1123-1136.

Neubauer, A.C., Grabner, R.H., Freudenthaler, H.H., Beckmann, J.F., Guthke, J., 2004. Intelligence and individual differences in becoming neurally efficient. Acta Psychol. (Amst.) 116 (1), 55-74.

Nielson, K.A., Langenecker, S.A., Garavan, H., 2002. Differences in the functional neuroanatomy of inhibitory control across the adult life span. Psychol. Aging 17 (1), 56-71.

Norman, D.A., Shallice, T., 1986. Attention to action: willed and automatic control of behavior. In: Davidson, R.J., Schwartz, G.E., Shapiro, D. (Eds.), Consciousness and Self-regulation, vol. 4. Plenum Press, New York, pp. 1-18.

Olesen, P.J., Westerberg, H., Klingberg, T., 2004. Increased prefrontal and parietal activity after training of working memory. Nat. Neurosci. 7 (1), $75-79$.

Parasuraman, R., Greenwood, P.M., Kumar, R., Fossella, J., 2005. Beyond heritability: neurotransmitter genes differentially modulate visuospatial attention and working memory. Psychol. Sci. 16 (3), 200-207.

Paulesu, E., Frith, C.D., Frackowiak, R.S., 1993. The neural correlates of the verbal component of working memory. Nature 362 (6418), $342-345$.

Poldrack, R.A., 2000. Imaging brain plasticity: conceptual and methodological issues-a theoretical review. NeuroImage 12 (1), 1-13.

Poldrack, R.A., Gabrieli, J.D., 2001. Characterizing the neural mechanisms of skill learning and repetition priming: evidence from mirror reading. Brain 124 (Pt. 1), 67-82.

Poldrack, R.A., Desmond, J.E., Glover, G.H., Gabrieli, J.D., 1998. The neural basis of visual skill learning: an fMRI study of mirror reading. Cereb. Cortex 8 (1), 1-10.

Posner, M.I., Petersen, S.E., 1990. The attention system of the human brain. Annu. Rev. Neurosci. 13, 25-42.

Raichle, M.E., Fiez, J.A., Videen, T.O., MacLeod, A.M., Pardo, J.V., Fox, P.T., Petersen, S.E., 1994. Practice-related changes in human brain functional anatomy during nonmotor learning. Cereb. Cortex 4 (1), $8-26$.

Raichle, M.E., MacLeod, A.M., Snyder, A.Z., Powers, W.J., Gusnard, D.A., Shulman, G.L., 2001. A default mode of brain function. Proc. Natl. Acad. Sci. U. S. A. 98 (2), 676-682.

Rainer, G., Miller, E.K., 2000. Effects of visual experience on the representation of objects in the prefrontal cortex. Neuron 27 (1), $179-189$.

Ridderinkhof, K.R., Ullsperger, M., Crone, E.A., Nieuwenhuis, S., 2004. The role of the medial frontal cortex in cognitive control. Science 306 (5695), 443-447.

Roberts, R.J., Hager, L.D., Heron, C., 1994. Prefrontal cognitive-processes-Working-memory and inhibition in the antisaccade task. J. Exp. Psychol. Gen. 123 (4), 374-393.

Rosenberg, D., Keshavan, M., Bagwell, W., Seymour, A., O'Hearn, K., Dick, E., Birmaher, B., Sweeney, J., 1996. Caudate nucleus and putamen abnormalities in pediatric OCD. Biol. Psychiatry 39 (7), 600.

Rubia, K., Russell, T., Bullmore, E.T., Soni, W., Brammer, M.J., Simmons, A., Taylor, E., Andrew, C., Giampietro, V., Sharma, T., 2001a. An fMRI study of reduced left prefrontal activation in schizophrenia during normal inhibitory function. Schizophr. Res. 52 (1-2), 47-55.

Rubia, K., Russell, T., Overmeyer, S., Brammer, M.J., Bullmore, E.T., Sharma, T., Simmons, A., Williams, S.C., Giampietro, V., Andrew, C.M., Taylor, E., 2001b. Mapping motor inhibition: conjunctive brain activations across different versions of go/no-go and stop tasks. NeuroImage 13 (2), 250-261.

Rubia, K., Smith, A.B., Brammer, M.J., Taylor, E., 2003. Right inferior prefrontal cortex mediates response inhibition while mesial prefrontal cortex is responsible for error detection. Neurolmage 20 (1), $351-358$.

Rueda, M.R., Rothbart, M.K., McCandliss, B.D., Saccomanno, L., Posner, M.I., 2005. Training, maturation, and genetic influences on the development of executive attention. Proc. Natl. Acad. Sci. U. S. A 102 (41), 14931-14936.

Rushworth, M.F.S., Hadland, K.A., Paus, T., Sipila, P.K., 2002. Role of the human medial frontal cortex in task switching: a combined fMRI and TMS study. J. Neurophysiol. 87 (5), 2577-2592.

Rypma, B., D'Esposito, M., 1999. The roles of prefrontal brain regions in components of working memory: effects of memory load and individual differences. Proc. Natl. Acad. Sci. U. S. A. 96 (11), $6558-6563$.

Rypma, B., Berger, J.S., D’Esposito, M., 2002. The influence of workingmemory demand and subject performance on prefrontal cortical activity. J. Cogn. Neurosci. 14 (5), $721-731$.

Sakai, K., Hikosaka, O., Miyauchi, S., Takino, R., Sasaki, Y., Putz, B., 1998. Transition of brain activation from frontal to parietal areas in visuomotor sequence learning. J. Neurosci. 18 (5), 1827-1840.

Sanes, J.N., Donoghue, J.P., 2000. Plasticity and primary motor cortex. Annu. Rev. Neurosci. 23, 393-415.

Schneider, W., Shiffrin, R.M., 1977. Controlled and automatic human information-processing: 1. Detection, search, and attention. Psychol. Rev. 84 (1), 1-66.

Schwartz, S., Maquet, P., Frith, C., 2002. Neural correlates of perceptual learning: a functional MRI study of visual texture discrimination. Proc. Natl. Acad. Sci. U. S. A. 99 (26), 17137-17142.

Shadmehr, R., Holcomb, H.H., 1997. Neural correlates of motor memory consolidation. Science 277 (5327), 821-825.

Shulman, G.L., Corbetta, M., Buckner, R.L., Raichle, M.E., Fiez, J.A., Miezin, F.M., Petersen, S.E., 1997a. Top-down modulation of early sensory cortex. Cereb. Cortex 7 (3), 193-206.

Shulman, G.L., Fiez, J.A., Corbetta, M., Buckner, R.L., Miezin, F.M., Raichle, M.E., Petersen, S.E., 1997b. Common blood flow changes across visual tasks: 2. Decreases in cerebral cortex. J. Cogn. Neurosci. 9 (5), 648-663.

Sigman, M., Pan, H., Yang, Y., Stern, E., Silbersweig, D., Gilbert, C.D., 2005. Top-down reorganization of activity in the visual pathway after learning a shape identification task. Neuron 46 (5), 823-835.

Smith, E.E., Jonides, J., 1998. Neuroimaging analyses of human working memory. Proc. Natl. Acad. Sci. 95 (20), 12061-12068.

Smith, E.E., Jonides, J., Marshuetz, C., Koeppe, R.A., 1998. Components of verbal working memory: evidence from neuroimaging. Proc. Natl. Acad. Sci. U. S. A. 95 (3), 876-882.

Staines, W.R., Graham, S.J., Black, S.E., McIlroy, W.E., 2002. Taskrelevant modulation of contralateral and ipsilateral primary somatosensory cortex and the role of a prefrontal-cortical sensory gating system. NeuroImage 15 (1), 190-199.

Talairach, J., Tournoux, P., 1988. Co-Planar Stereotaxic Atlas of the Human Brain. Thieme, New York.

Troyer, A.K., Black, S.E., Armilio, M.L., Moscovitch, M., 2004. Cognitive and motor functioning in a patient with selective infarction of the left basal ganglia: evidence for decreased nonroutine response selection and performance. Neuropsychologia 42 (7), 902-911.

Ullsperger, M., Von Cramon, D.Y., 2003. Error monitoring using external feedback: specific roles of the habenular complex, the reward system, 
and the cingulate motor area revealed by functional magnetic resonance imaging. J. Neurosci. 23 (10), 4308-4314.

Unsworth, N., Schrock, J.C., Engle, R.W., 2004. Working memory capacity and the antisaccade task: individual differences in voluntary saccade control. J. Exper. Psychol., Learn., Mem., Cogn. 30 (6), $1302-1321$.

Visscher, K.M., Miezin, F.M., Kelly, J.E., Buckner, R.L., Donaldson, D.I., McAvoy, M.P., Bhalodia, V.M., Petersen, S.E., 2003. Mixed blocked/event-related designs separate transient and sustained activity in fMRI. NeuroImage 19 (4), 1694-1708.

Wager, T.D., Smith, E.E., 2003. Neuroimaging studies of working memory: a meta-analysis. Cogn. Affect. Behav. Neurosci. 3 (4), 255-274.

Watanabe, J., Sugiura, M., Sato, K., Sato, Y., Maeda, Y., Matsue, Y., Fukuda, H., Kawashima, R., 2002. The human prefrontal and parietal association cortices are involved in NO-GO performances: an eventrelated fMRI study. NeuroImage 17 (3), 1207-1216. 